\title{
Total Synthesis and Anti-Tobacco Mosaic Virus Activity of the Furofuran Lignan (士)-Phrymarolin II and Its Analogues
}

\author{
Supporting Information \\ Yuan Chi, ${ }^{\dagger}$ Huan Zhou, ${ }^{\dagger}$ Hong-Wei He, Yi-Dan Ma, Bo Li, Dan Xu, \\ Jin-Ming $\mathrm{GaO}^{*}$ and Gong $\mathrm{Xu}^{*}$ \\ ${ }^{\dagger}$ Y. Chi and H. Zhou contributed equally
}

Table of Contents

The Chan-Lam-Evans cross-coupling reaction of ( \pm -24 under different conditions SI-2

Comparison of the ${ }^{1} \mathrm{H}$ and ${ }^{13} \mathrm{C}$ NMR data of the synthesized ( \pm )-phrymarolin II with literature data SI-3

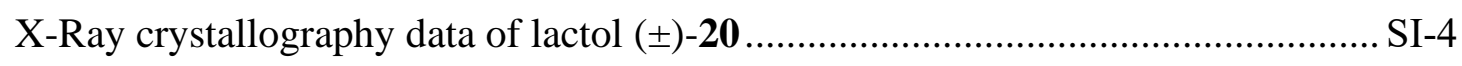

NMR spectra SI-7 
Table S1. The Chan-Lam-Evans cross-coupling reaction of ( \pm )-24 under different conditions. $^{a}$

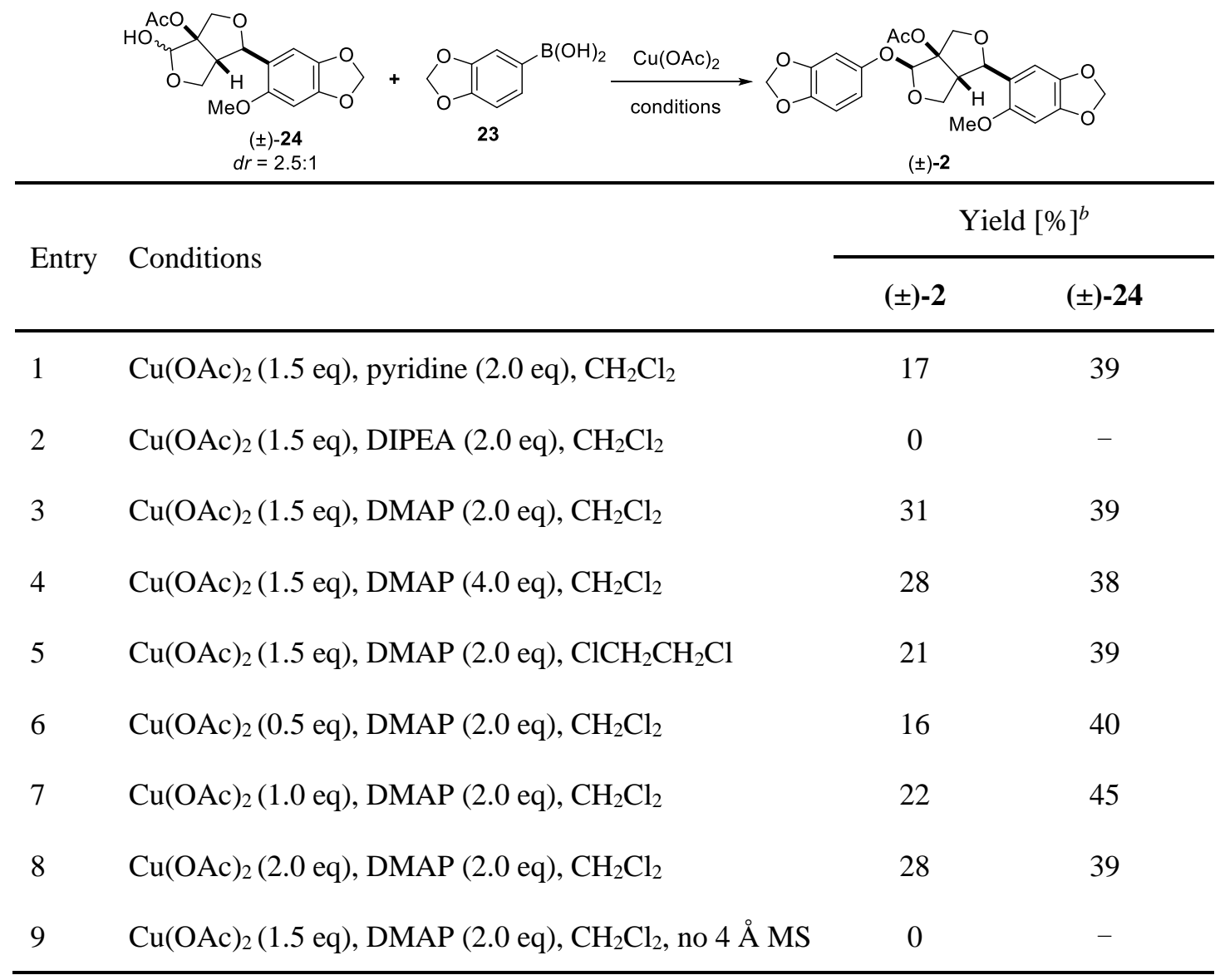

${ }^{a}$ Reaction conditions: compound ( \pm )-24 (150 mg, $0.443 \mathrm{mmol}, 1.0$ equiv), compound 23 (220 mg, $1.33 \mathrm{mmol}, 3.0$ equiv), $4 \AA$ molecular sieves $(450 \mathrm{mg})$, solvent $(9.0 \mathrm{~mL}), 40{ }^{\circ} \mathrm{C}$, under $\mathrm{O}_{2}$ atmosphere for $24 \mathrm{~h} .{ }^{b}$ Isolated yield. 
Table S2. Comparison of the ${ }^{1} \mathrm{H}$ and ${ }^{13} \mathrm{C}$ NMR data of the synthesized ( \pm )phrymarolin II with literature data.

\begin{tabular}{|c|c|c|c|c|}
\hline \multirow[b]{2}{*}{ position } & \multicolumn{2}{|r|}{ natural phrymarolin $\mathrm{II}^{a}$} & \multicolumn{2}{|r|}{ synthetic $( \pm)$-phrymarolin II $^{b}$} \\
\hline & $\delta_{\mathrm{C}}$ & $\delta_{\mathrm{H}}$ & $\delta_{\mathrm{C}}$ & $\delta_{\mathrm{H}}$ \\
\hline 1 & 96.6 & & 96.5 & \\
\hline 2 & 101.4 & $5.70(\mathrm{~s}, 1 \mathrm{H})$ & 101.4 & $(\mathrm{~s}, 1 \mathrm{H})$ \\
\hline \multirow[t]{2}{*}{4} & 68.8 & $4.06(\mathrm{dd}, J=9.2,1.5 \mathrm{~Hz}, 1 \mathrm{H})$ & 68.7 & $4.06(\mathrm{dd}, J=9.2,1.9 \mathrm{~Hz}, 1 \mathrm{H})$ \\
\hline & & $4.29(\mathrm{dd}, J=9.1,6.9 \mathrm{~Hz}, 1 \mathrm{H})$ & & $4.29 \quad(\mathrm{dd}, J=9.2,7.0 \mathrm{~Hz}, 1 \mathrm{H})$ \\
\hline 5 & 56.7 & $2.84(\mathrm{t}, J=6.6 \mathrm{~Hz}, 1 \mathrm{H})$ & 56.6 & $2.84(\mathrm{t}, J=7.0 \mathrm{~Hz}, 1 \mathrm{H})$ \\
\hline 6 & 83.1 & $4.87(\mathrm{~d}, J=7.0 \mathrm{~Hz}, 1 \mathrm{H})$ & 83.0 & $4.87(\mathrm{~d}, J=7.1 \mathrm{~Hz}, 1 \mathrm{H})$ \\
\hline \multirow[t]{2}{*}{8} & 75.8 & $3.81(\mathrm{~d}, J=11.1 \mathrm{~Hz}, 1 \mathrm{H})$ & 75.7 & $3.81(\mathrm{~d}, J=11.3 \mathrm{~Hz}, 1 \mathrm{H})$ \\
\hline & & $4.60(\mathrm{~d}, J=11.1 \mathrm{~Hz}, 1 \mathrm{H})$ & & $4.60(\mathrm{~d}, J=11.1 \mathrm{~Hz}, 1 \mathrm{H})$ \\
\hline 9 & 152.0 & & 151.9 & \\
\hline 10 & 106.5 & $6.58(\mathrm{~d}, J=2.3 \mathrm{~Hz}, 1 \mathrm{H})$ & 106.4 & $6.58(\mathrm{~d}, J=2.2 \mathrm{~Hz}, 1 \mathrm{H})$ \\
\hline 11 & 148.2 & & 148.1 & \\
\hline 12 & 141.6 & & 141.5 & \\
\hline 13 & 108.2 & $6.69(\mathrm{~d}, J=8.4 \mathrm{~Hz}, 1 \mathrm{H})$ & 108.1 & $6.69(\mathrm{~d}, J=8.2 \mathrm{~Hz}, 1 \mathrm{H})$ \\
\hline 14 & 110.6 & $6.49(\mathrm{dd}, J=8.4,2.3 \mathrm{~Hz}, 1 \mathrm{H})$ & 110.5 & $6.49(\mathrm{dd}, J=8.6,2.2 \mathrm{~Hz}, 1 \mathrm{H})$ \\
\hline 15 & 101.4 & $5.93(\mathrm{~s}, 2 \mathrm{H})$ & 101.4 & $5.93 \quad(\mathrm{~s}, 2 \mathrm{H})$ \\
\hline $9^{\prime}$ & 121.5 & & 121.4 & \\
\hline $10^{\prime}$ & 103.2 & $7.05(\mathrm{~s}, 1 \mathrm{H})$ & 103.1 & $7.05(\mathrm{~s}, 1 \mathrm{H})$ \\
\hline $11^{\prime}$ & 143.5 & & 143.4 & \\
\hline $12^{\prime}$ & 147.7 & & 147.6 & \\
\hline $13^{\prime}$ & 94.4 & $6.52(\mathrm{~s}, 1 \mathrm{H})$ & 94.3 & $6.52(\mathrm{~s}, 1 \mathrm{H})$ \\
\hline $14^{\prime}$ & 151.6 & & 151.5 & \\
\hline $15^{\prime}$ & 101.4 & $5.92(\mathrm{~s}, 2 \mathrm{H})$ & 101.3 & $5.91(\mathrm{~s}, 2 \mathrm{H})$ \\
\hline $\mathrm{MeO}-$ & 56.4 & $3.77(\mathrm{~s}, 3 \mathrm{H})$ & 56.3 & $3.77 \quad(\mathrm{~s}, 3 \mathrm{H})$ \\
\hline \multirow[t]{2}{*}{ AcO- } & 170.9 & & 170.9 & \\
\hline & 21.2 & $2.13(\mathrm{~s}, 3 \mathrm{H})$ & 21.2 & $2.13(\mathrm{~s}, 3 \mathrm{H})$ \\
\hline
\end{tabular}

${ }^{a}$ Data obtained from the literature (Y. Li, J. Wei, J. Fang, W. Lv, Y. Ji, A. A. A. Aioub, J. Zhang, Z. $\mathrm{Hu}$, Molecules 2019, 24, 1976) were recorded in $\mathrm{CDCl}_{3}$ at $500 \mathrm{MHz}$ for ${ }^{1} \mathrm{H} \mathrm{NMR}$ and $126 \mathrm{MHz}$ for ${ }^{13} \mathrm{C}$ NMR. ${ }^{b}$ Data recorded in $\mathrm{CDCl}_{3}$ at $400 \mathrm{MHz}$ for ${ }^{1} \mathrm{H}$ NMR and $101 \mathrm{MHz}$ for ${ }^{13} \mathrm{C}$ NMR. 


\section{X-Ray Crystallography Data of Lactol ( \pm )-20}

Single crystal of lactol ( \pm )-20 was obtained by recrystallization from dichloromethane and ethyl acetate. The structure is shown in Figure S1. X-ray diffraction data and the refinement are shown in Table S3-4. CIF file of $( \pm)-20$ can be obtained from the Cambridge Crystallographic Data Center using deposition number CCDC: 2053387.

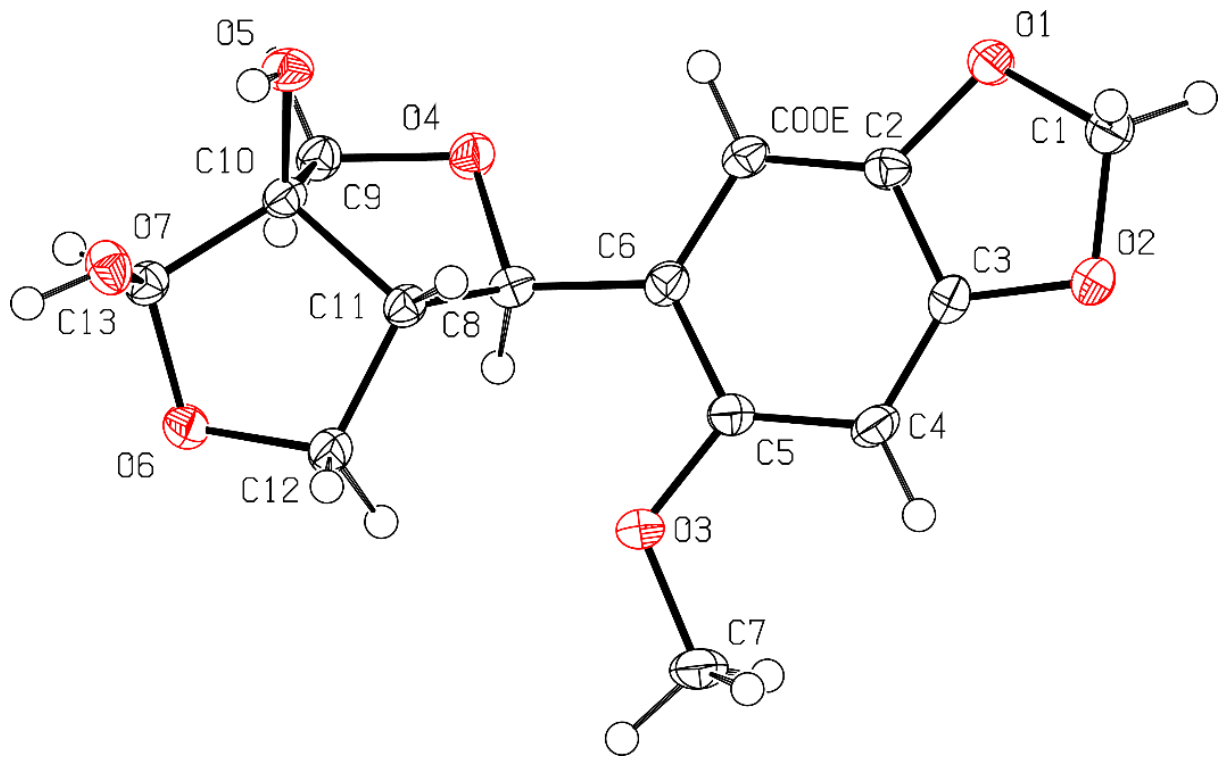

Figure S1. X-ray single crystal structure of lactol $( \pm)$-20

(Displacement ellipsoids are drawn at the $50 \%$ probability level)

Table S3. Experimental details for lactol ( \pm )-20.

\begin{tabular}{|c|c|}
\hline & tianyu20200928_1_0m \\
\hline \multicolumn{2}{|l|}{ Crystal data } \\
\hline Chemical formula & $\mathrm{C}_{14} \mathrm{H}_{16} \mathrm{O}_{7}$ \\
\hline$M_{\mathrm{r}}$ & 296.27 \\
\hline $\begin{array}{l}\text { Crystal system, space } \\
\text { group }\end{array}$ & Monoclinic, $P 2_{1} / c$ \\
\hline Temperature (K) & 153 \\
\hline$a, b, c(\AA)$ & 20.2746 (7), 5.3528 (2), 11.9234 (4) \\
\hline$\beta\left(^{\circ}\right)$ & 95.579 (1) \\
\hline
\end{tabular}




\begin{tabular}{|c|c|}
\hline$V\left(\AA^{3}\right)$ & $1287.87(8)$ \\
\hline$Z$ & 4 \\
\hline Radiation type & Synchrotron, $\lambda=1.54184 \AA$ \\
\hline$\mu\left(\mathrm{mm}^{-1}\right)$ & 1.06 \\
\hline Crystal size (mm) & $0.1 \times 0.1 \times 0.05$ \\
\hline \multicolumn{2}{|l|}{ Data collection } \\
\hline Diffractometer & $\begin{array}{l}\text { Bruker APEX-II CCD } \\
\text { diffractometer }\end{array}$ \\
\hline Absorption correction & Multi-scan \\
\hline$T_{\min }, T_{\max }$ & $0.546,0.753$ \\
\hline $\begin{array}{l}\text { No. of measured, } \\
\text { independent and } \\
\text { observed }[I>2 \sigma(I)] \\
\text { reflections }\end{array}$ & $22526,2337,2279$ \\
\hline$R_{\text {int }}$ & 0.040 \\
\hline$(\sin \theta / \lambda)_{\max }\left(\AA^{-1}\right)$ & 0.603 \\
\hline \multicolumn{2}{|l|}{ Refinement } \\
\hline $\begin{array}{l}R\left[F^{2}>2 \sigma\left(F^{2}\right)\right] \\
w R\left(F^{2}\right), S\end{array}$ & $0.035, \quad 0.089, \quad 1.10$ \\
\hline No. of reflections & 2337 \\
\hline No. of parameters & 194 \\
\hline $\mathrm{H}$-atom treatment & H-atom parameters constrained \\
\hline$\left.\Delta\rangle_{\max }, \Delta\right\rangle_{\min }\left(\mathrm{e} \AA^{-3}\right)$ & $0.31,-0.20$ \\
\hline
\end{tabular}

Computer programs: SAINT V8.40A (Bruker, 2016), SHELXT 2018/2 (Sheldrick, 2018), SHELXL 2018/3 (Sheldrick, 2015), Olex2 1.3-alpha (Dolomanov et al., 2009). 
Table S4. Selected geometric parameters ( $)$ for lactol ( \pm )-20.

\begin{tabular}{|l|l|l|l|}
\hline $\mathrm{O} 1-\mathrm{C} 1$ & $1.4332(16)$ & $\mathrm{C} 3-\mathrm{C} 4$ & $1.3749(19)$ \\
\hline $\mathrm{O} 1-\mathrm{C} 2$ & $1.3918(16)$ & $\mathrm{C} 4-\mathrm{H} 4$ & 0.9500 \\
\hline $\mathrm{O} 2-\mathrm{C} 1$ & $1.4338(17)$ & $\mathrm{C} 4-\mathrm{C} 5$ & $1.3994(19)$ \\
\hline $\mathrm{O} 2-\mathrm{C} 3$ & $1.3828(16)$ & $\mathrm{C} 5-\mathrm{C} 6$ & $1.4015(18)$ \\
\hline $\mathrm{O} 3-\mathrm{C} 5$ & $1.3690(17)$ & $\mathrm{C} 6-\mathrm{C} 8$ & $1.5050(18)$ \\
\hline $\mathrm{O} 3-\mathrm{C} 7$ & $1.4193(17)$ & $\mathrm{C} 7-\mathrm{H} 7 \mathrm{~A}$ & 0.9800 \\
\hline $\mathrm{O} 4-\mathrm{C} 8$ & $1.4350(15)$ & $\mathrm{C} 7-\mathrm{H} 7 \mathrm{~B}$ & 0.9800 \\
\hline $\mathrm{O} 4-\mathrm{C} 9$ & $1.4317(15)$ & $\mathrm{C} 7-\mathrm{H} 7 \mathrm{C}$ & 0.9800 \\
\hline $\mathrm{O} 5-\mathrm{H} 5$ & 0.8400 & $\mathrm{C} 8-\mathrm{H} 8$ & 1.0000 \\
\hline $\mathrm{O} 5-\mathrm{C} 10$ & $1.4153(16)$ & $\mathrm{C} 8-\mathrm{C} 11$ & $1.5545(17)$ \\
\hline $\mathrm{O} 6-\mathrm{C} 12$ & $1.4630(15)$ & $\mathrm{C} 9-\mathrm{H} 9 \mathrm{~A}$ & 0.9900 \\
\hline $\mathrm{O} 6-\mathrm{C} 13$ & $1.4240(16)$ & $\mathrm{C} 9-\mathrm{H} 9 \mathrm{~B}$ & 0.9900 \\
\hline $\mathrm{O} 7-\mathrm{H} 7$ & 0.8400 & $\mathrm{C} 9-\mathrm{C} 10$ & $1.5242(17)$ \\
\hline $\mathrm{O} 7-\mathrm{C} 13$ & $1.3978(16)$ & $\mathrm{C} 10-\mathrm{C} 11$ & $1.5539(16)$ \\
\hline $\mathrm{C} 00 \mathrm{E}-\mathrm{H} 00 \mathrm{E}$ & 0.9500 & $\mathrm{C} 10-\mathrm{C} 13$ & $1.5341(17)$ \\
\hline $\mathrm{C} 00 \mathrm{E}-\mathrm{C} 2$ & $1.3790(18)$ & $\mathrm{C} 11-\mathrm{H} 11$ & 1.0000 \\
\hline $\mathrm{C} 00 \mathrm{E}-\mathrm{C} 6$ & $1.4003(19)$ & $\mathrm{C} 11-\mathrm{C} 12$ & $1.5295(18)$ \\
\hline $\mathrm{C} 1-\mathrm{H} 1 \mathrm{~A}$ & 0.9900 & $\mathrm{C} 12-\mathrm{H} 12 \mathrm{~A}$ & 0.9900 \\
\hline $\mathrm{C} 1-\mathrm{H} 1 \mathrm{~B}$ & 0.9900 & $\mathrm{C} 12-\mathrm{H} 12 \mathrm{~B}$ & 0.9900 \\
\hline $\mathrm{C} 2-\mathrm{C} 3$ & $1.3774(18)$ & $\mathrm{C} 13-\mathrm{H} 13$ & 1.0000 \\
\hline & & & \\
\hline
\end{tabular}




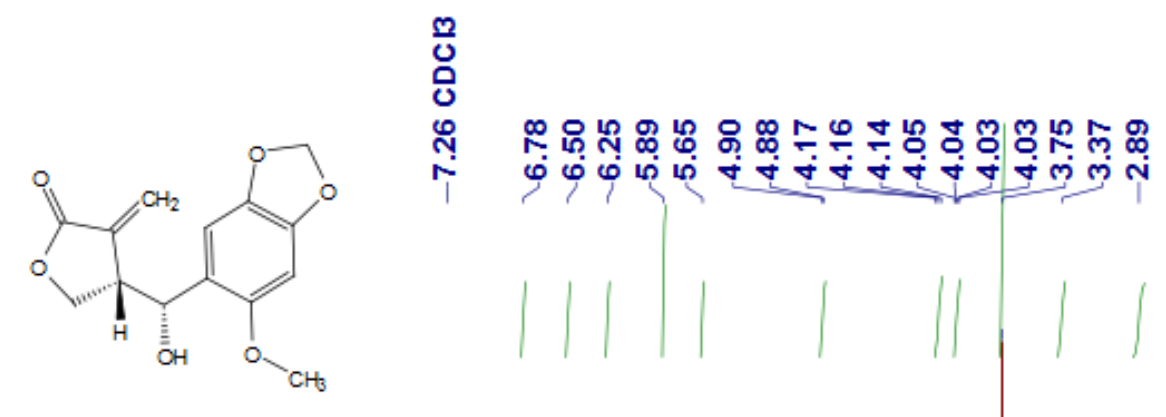

Alcohol ( \pm )-26

${ }^{1} \mathrm{H}$ NMR

(500 MHz, $\mathrm{CDCl}_{3} / \mathrm{TMS}$ )

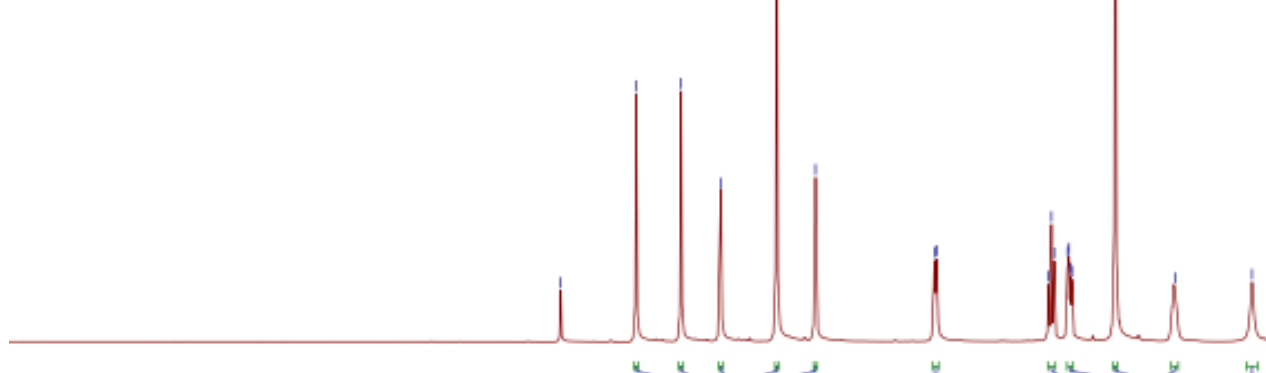

TMS

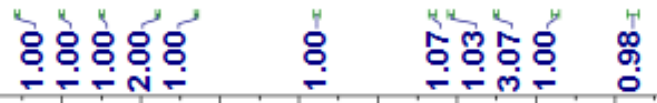

$\begin{array}{lllllllllllllllllllllll}10.5 & 10.0 & 9.5 & 9.0 & 8.5 & 8.0 & 7.5 & 7.0 & 6.5 & 6.0 & 5.5 & \begin{array}{c}5.0 \\ \mathrm{f} 1(\mathrm{ppm})\end{array} & 4.5 & 4.0 & 3.5 & 3.0 & 2.5 & 2.0 & 1.5 & 1.0 & 0.5 & 0.0 & -0.5\end{array}$

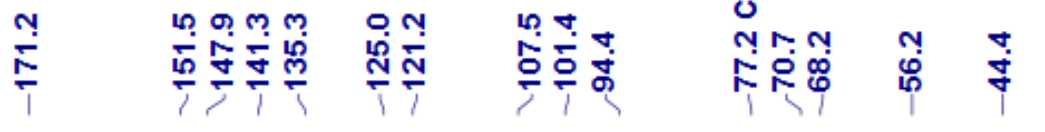

$\stackrel{\circ}{i}$<smiles>C=C1C(=O)OC[C@H]1[C@H](O)c1cc2c(cc1OC)OCO2</smiles>

Alcohol ( \pm -26

${ }^{13} \mathrm{C}$ NMR

(126 MHz, $\mathrm{CDCl}_{3} / \mathrm{TMS}$ )

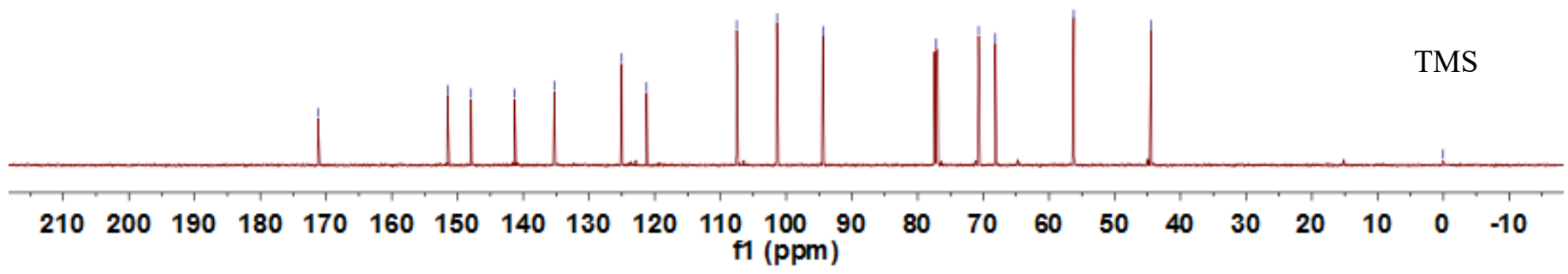




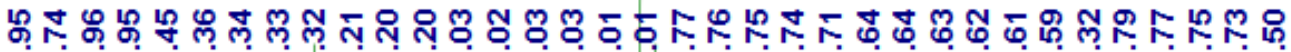
ம
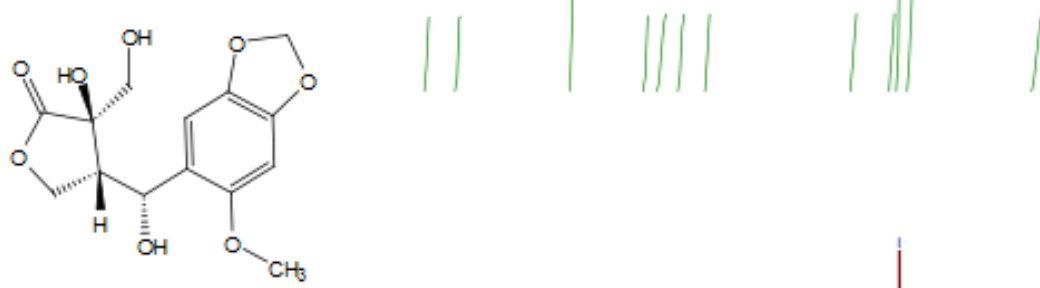

Triol ( \pm -29

${ }^{1} \mathrm{H}$ NMR

$\left(500 \mathrm{MHz}, \mathrm{DMSO}-d_{6}\right)$

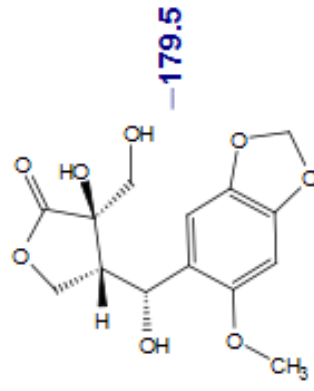

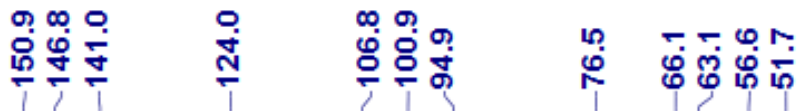

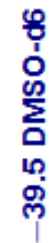

Triol ( \pm -29

${ }^{13} \mathrm{C}$ NMR

(126 MHz, DMSO- $\left.d_{6}\right)$

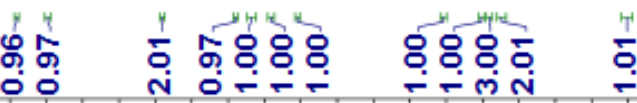

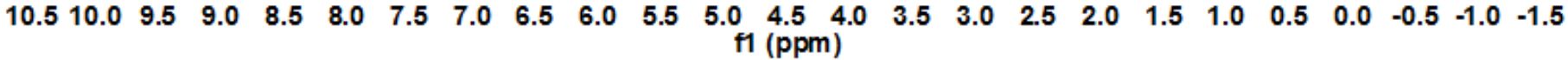

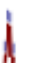




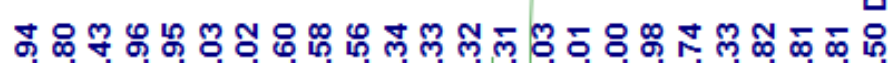
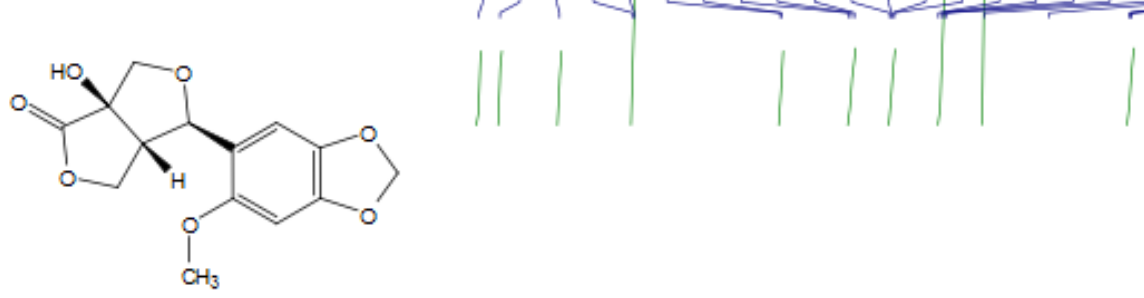

$$
\begin{gathered}
\text { Lactone }( \pm)-25 \\
{ }^{1} \mathrm{H} \text { NMR } \\
\left(500 \mathrm{MHz}, \mathrm{DMSO}-d_{6}\right)
\end{gathered}
$$

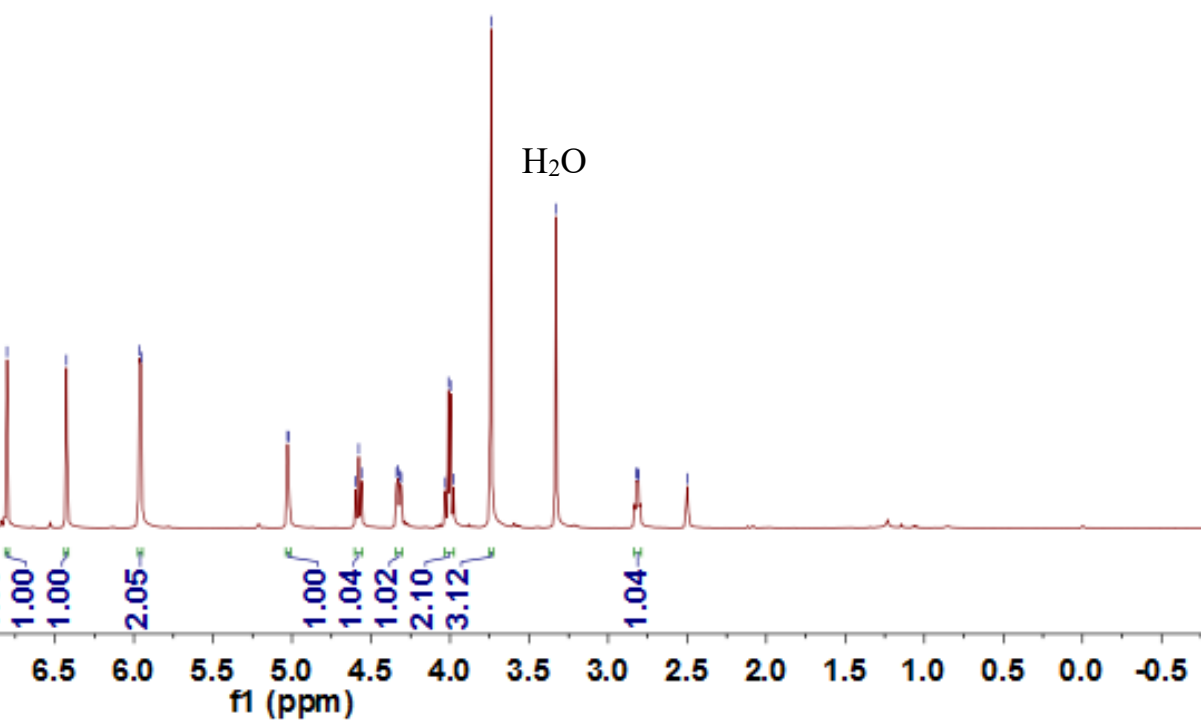

$\begin{array}{lllllllllllllllllllllll}10.5 & 10.0 & 9.5 & 9.0 & 8.5 & 8.0 & 7.5 & 7.0 & 6.5 & 6.0 & \begin{array}{l}5.5 \\ \mathrm{f} 1\end{array} \underset{(\mathrm{ppm})}{5.0} & 4.5 & 4.0 & 3.5 & 3.0 & 2.5 & 2.0 & 1.5 & 1.0 & 0.5 & 0.0 & -0.5\end{array}$

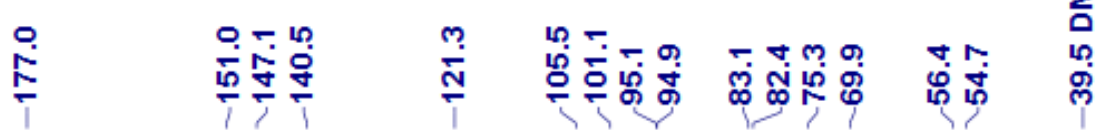

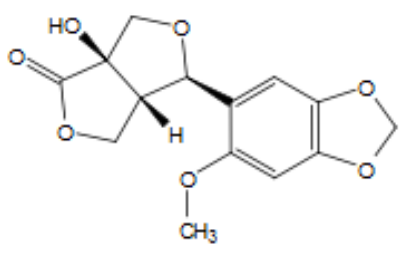

Lactone $( \pm)-25$

${ }^{13} \mathrm{C}$ NMR

(126 MHz, DMSO- $d_{6}$ )

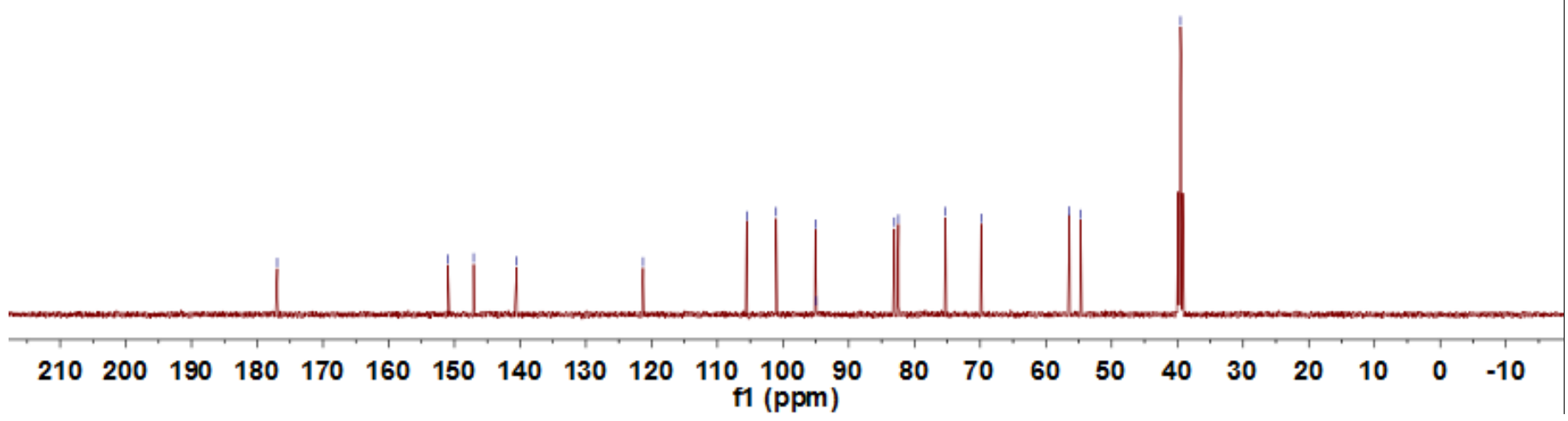




\section{象}

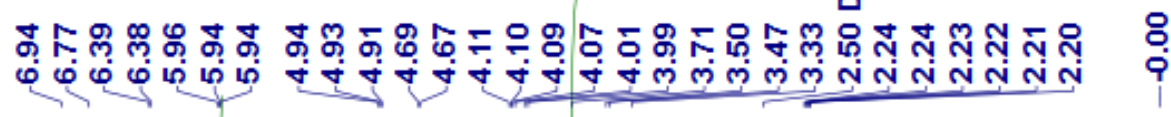

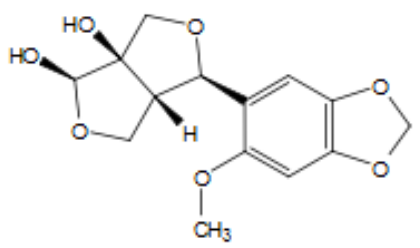

Lactol ( \pm )-20

${ }^{1} \mathrm{H}$ NMR

(400 MHz, DMSO- $d_{6} / \mathrm{TMS}$ )

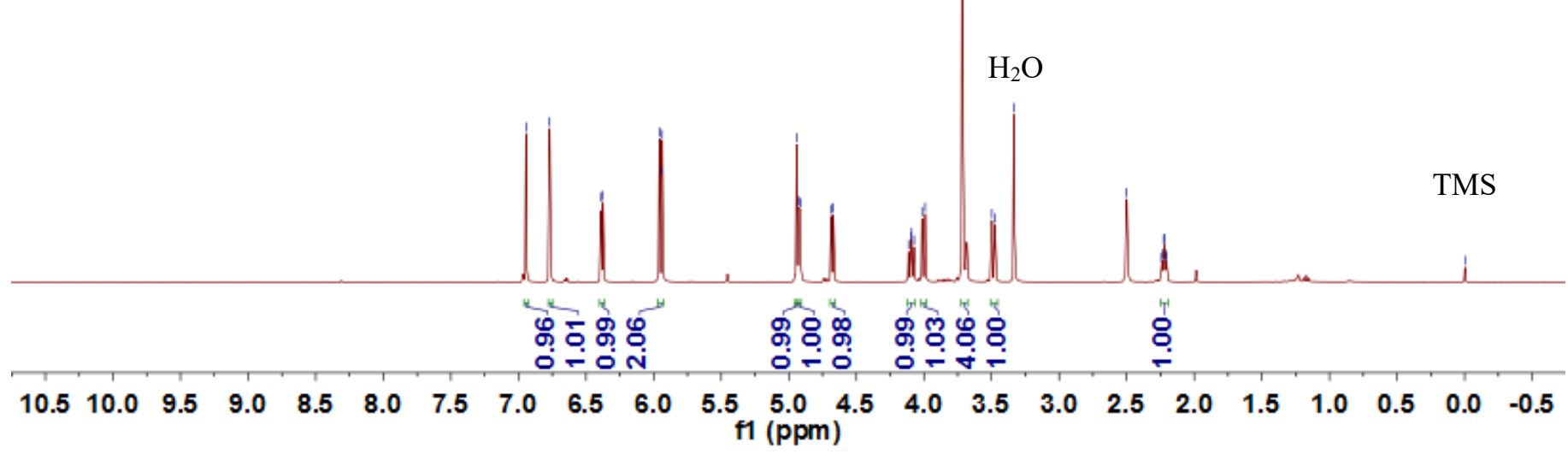

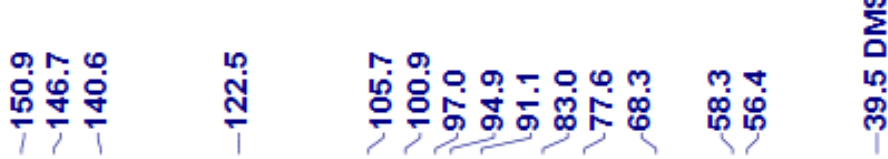
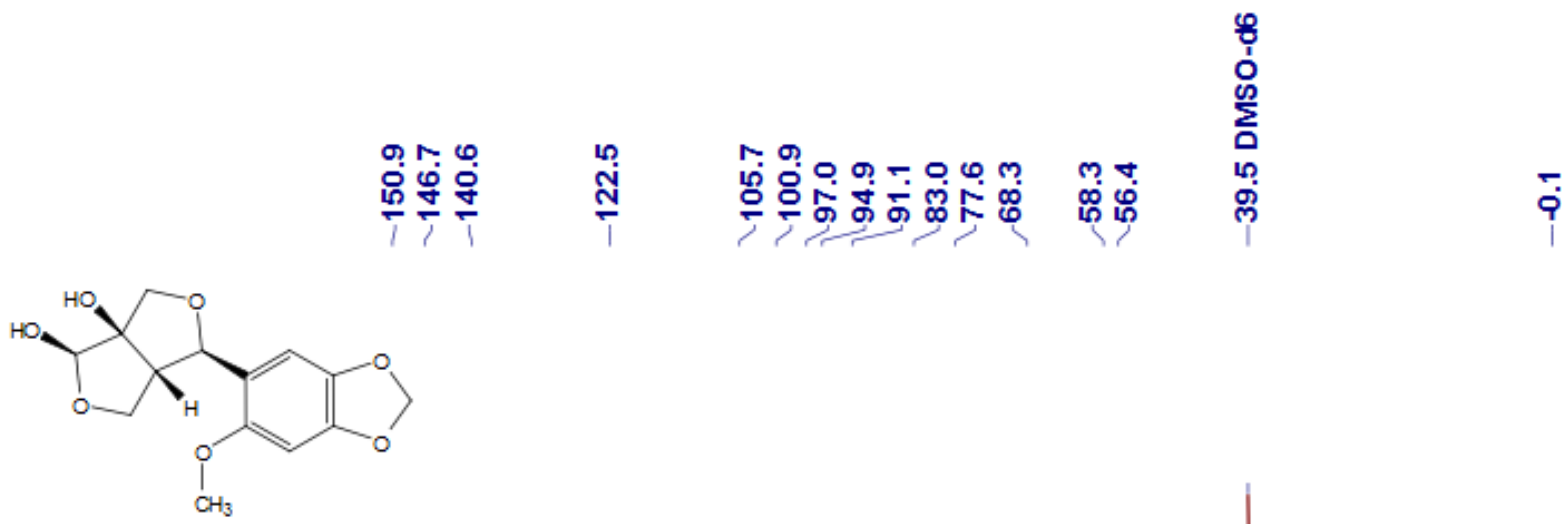

Lactol $( \pm)-20$

${ }^{13} \mathrm{C}$ NMR

(101 MHz, DMSO-d $\left.d_{6} / \mathrm{TMS}\right)$

TMS

$\begin{array}{lllllllllllllllllllllll}210 & 200 & 190 & 180 & 170 & 160 & 150 & 140 & 130 & 120 & 110 & 100 & 90 & 80 & 70 & 60 & 50 & 40 & 30 & 20 & 10 & 0 & -10\end{array}$

f1 (ppm) 


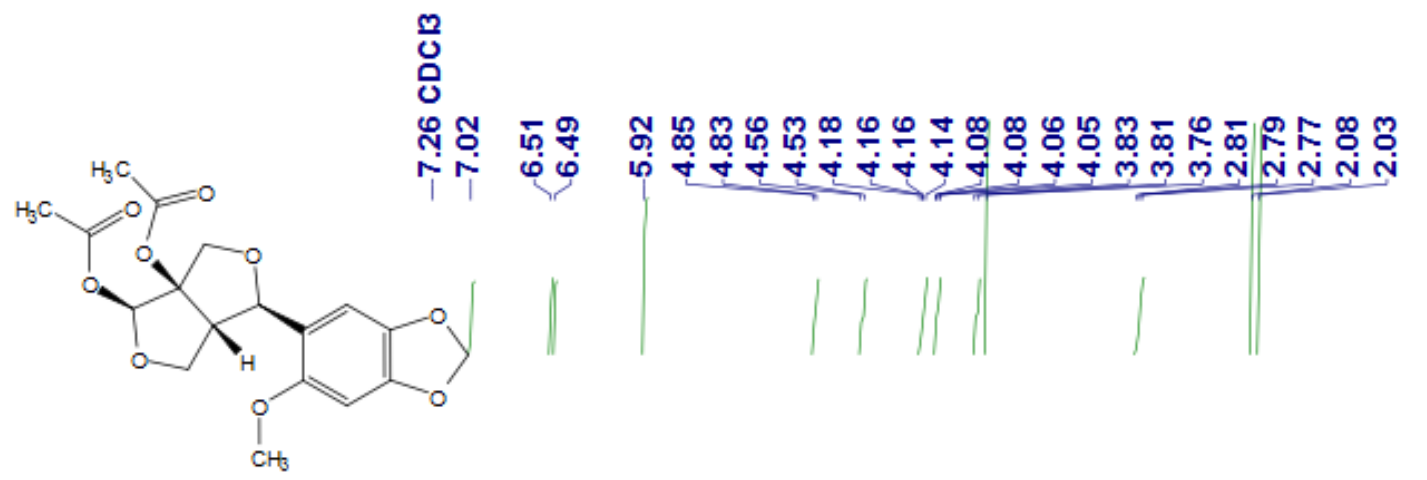

চ઼.

Diacetate ( $( \pm)-\mathbf{3 0}$

${ }^{1} \mathrm{H}$ NMR

(400 MHz, $\mathrm{CDCl}_{3} / \mathrm{TMS}$ )

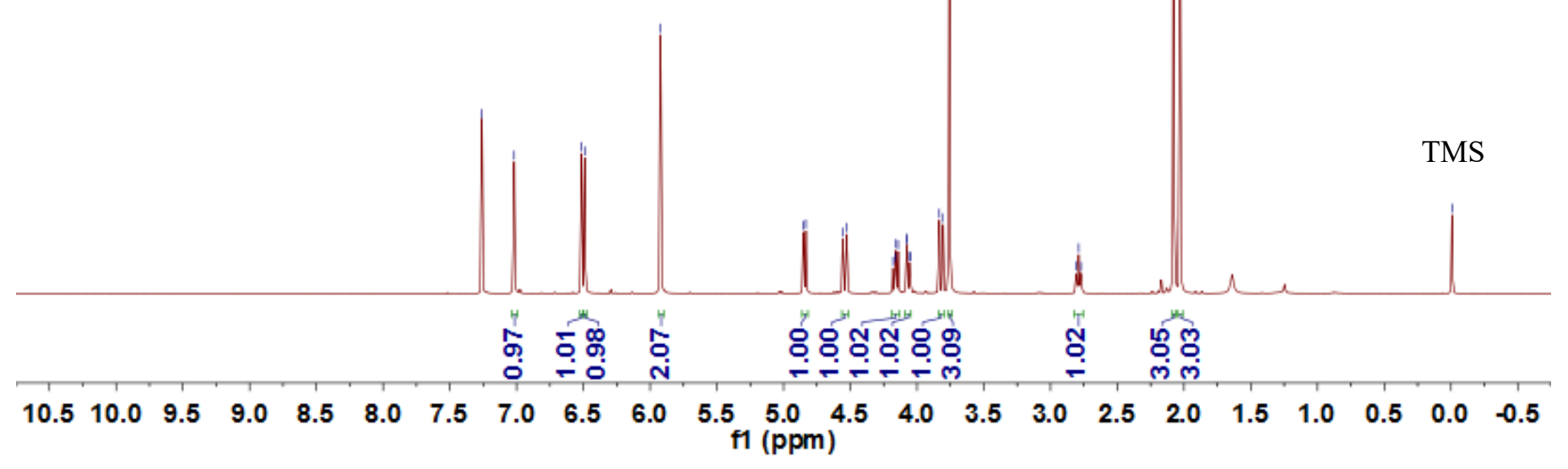

웅

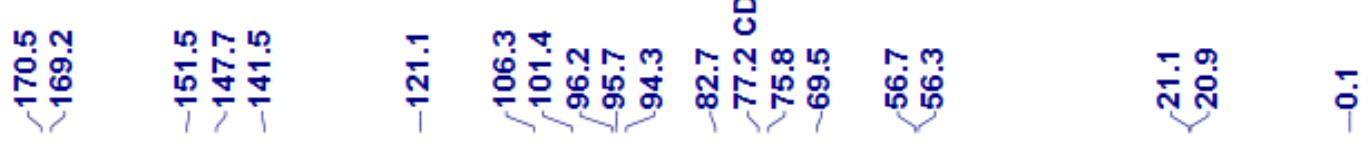<smiles>[2H][C@]1(O)[C@@H]2OC[C@H](OC(C)=O)[C@@]1(OC(C)=O)COC2c1cc2c(cc1OC)OCO2</smiles>

Diacetate ( \pm )-30

${ }^{13} \mathrm{C}$ NMR

(101 MHz, $\left.\mathrm{CDCl}_{3} / \mathrm{TMS}\right)$

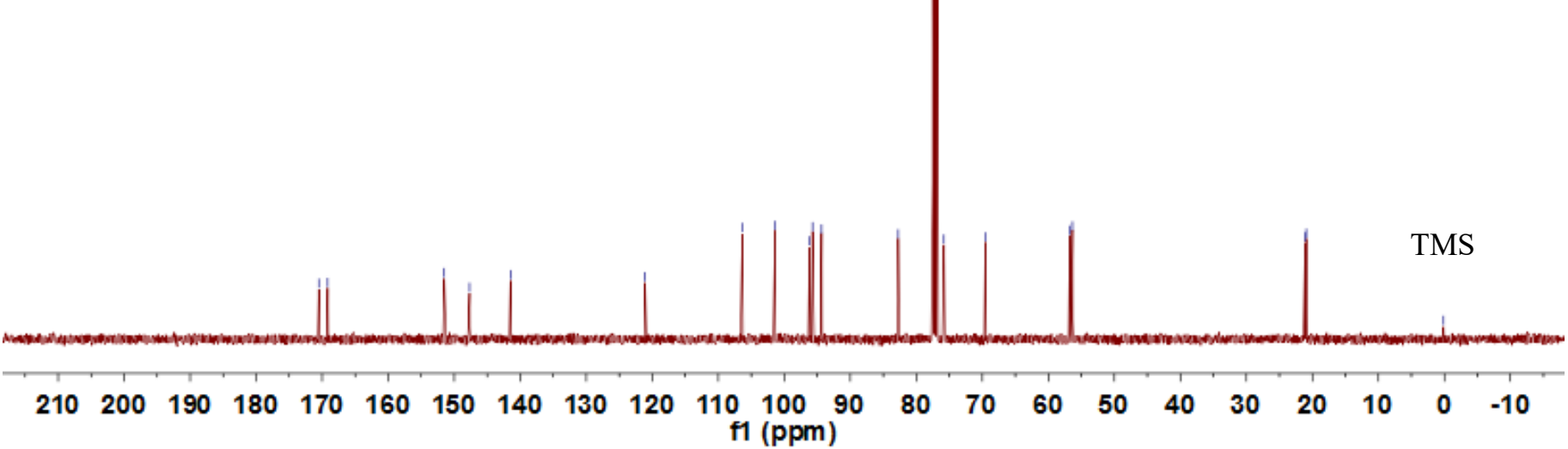


응<smiles>COc1cc2c(cc1[C@@H]1OC[C@]3(OC(C)=O)[C@@H](O)OC[C@H]13)OCO2</smiles>

ஸุ

Lactol ( \pm )-24

${ }^{1} \mathrm{H}$ NMR

(400 MHz, $\mathrm{CDCl}_{3} / \mathrm{TMS}$ )

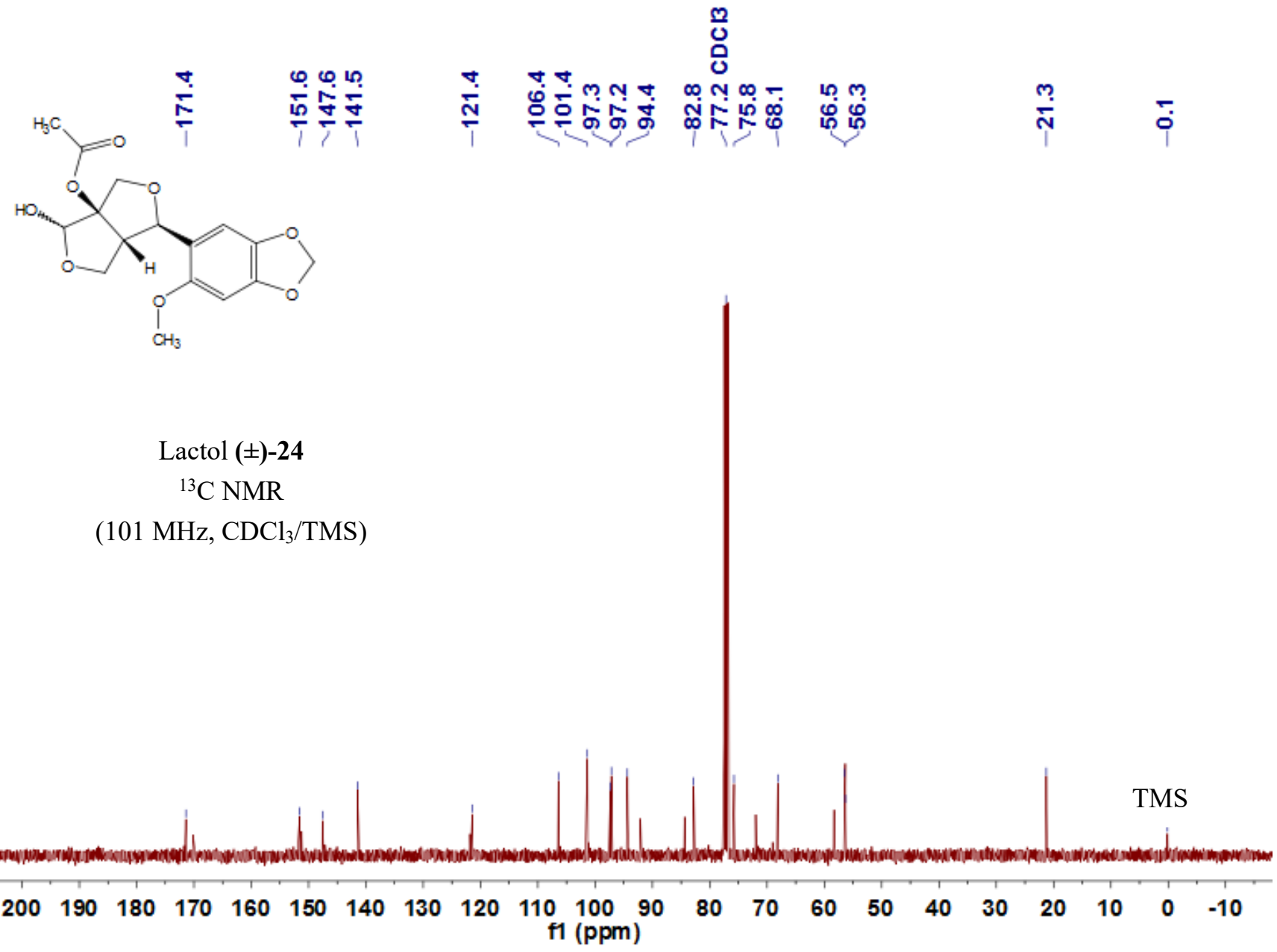

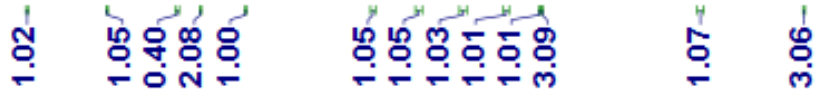

은

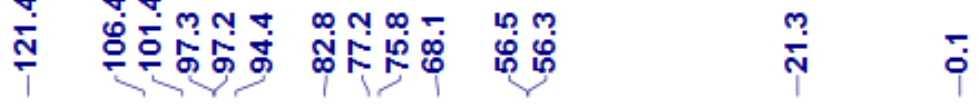

TMS

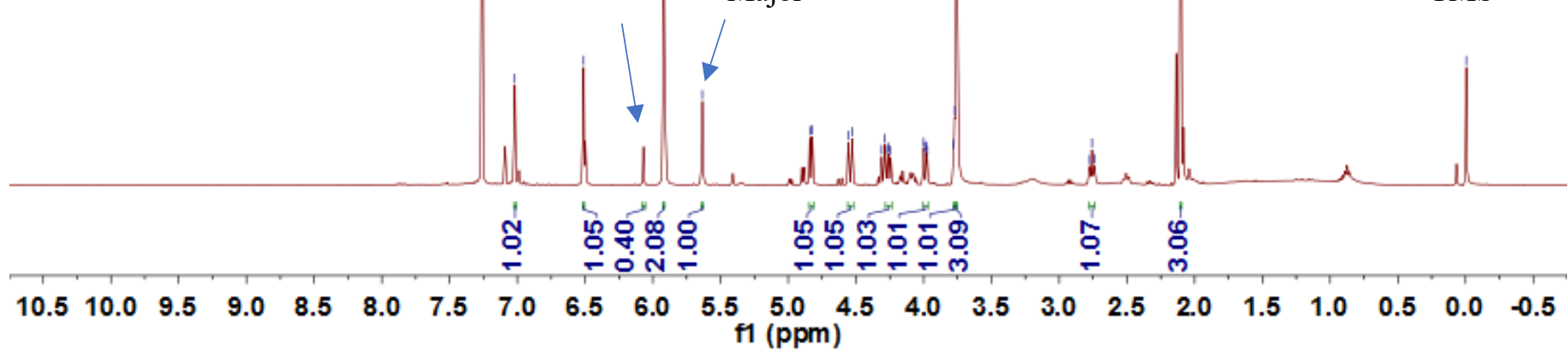




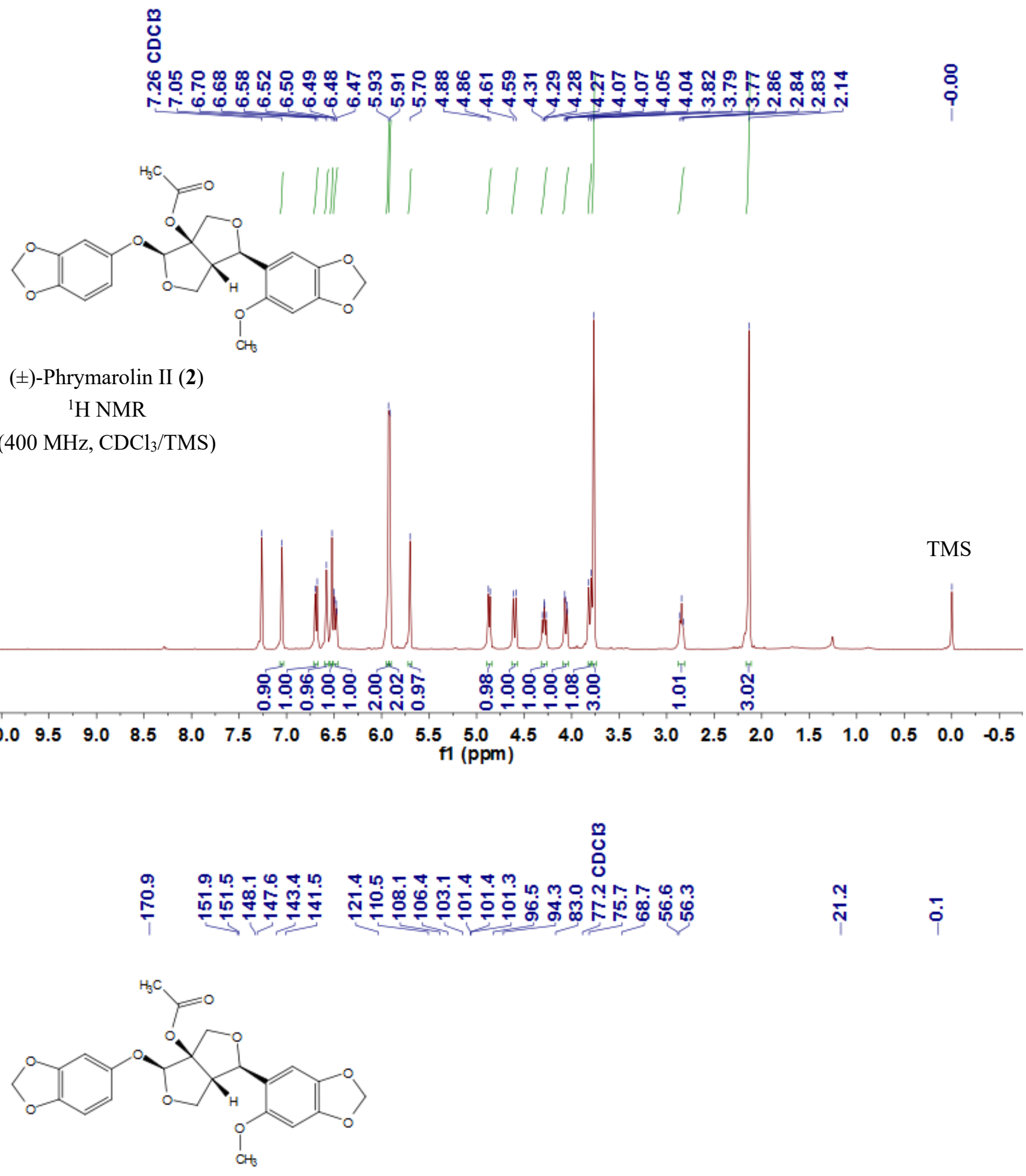
( \pm )-Phrymarolin II (2)
${ }^{13} \mathrm{C}$ NMR

(101 MHz, $\mathrm{CDCl}_{3} / \mathrm{TMS}$ )

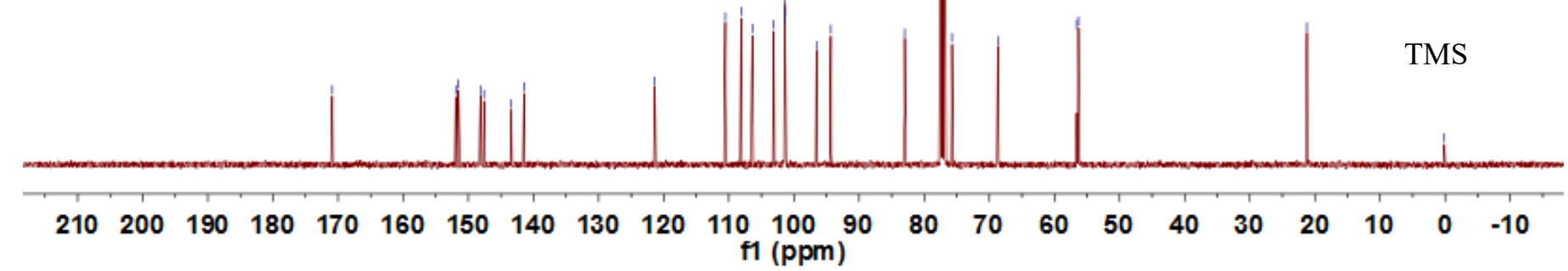


응

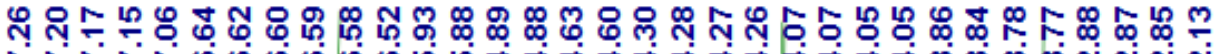

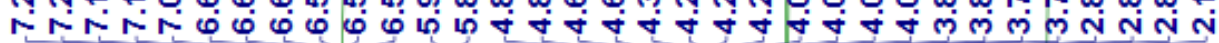<smiles>C=CC#CC1OC[C@]2(OC(C)=O)[C@@H](c3cc4c(cc3OC)OCO4)OC[C@H]1[C@H]2Oc1cccc(OC)c1</smiles>

$( \pm)-31$ a

${ }^{1} \mathrm{H}$ NMR

(400 MHz, $\mathrm{CDCl}_{3} / \mathrm{TMS}$ )

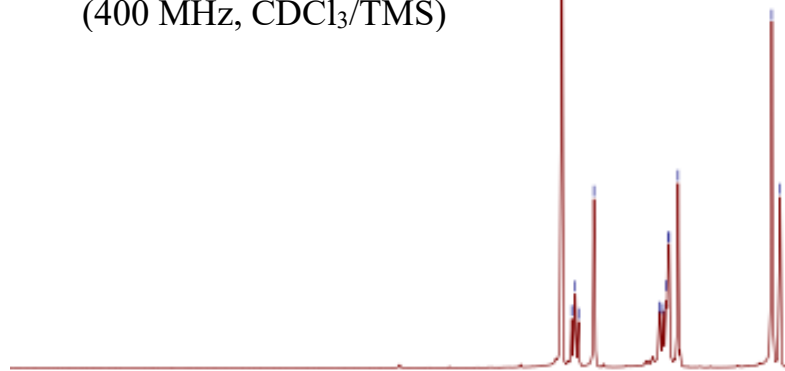

Non

TMS

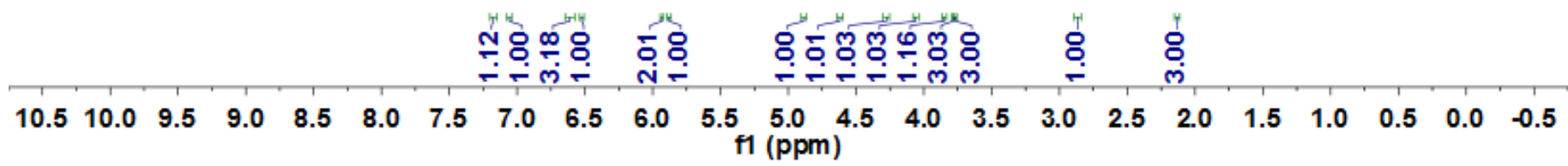

号

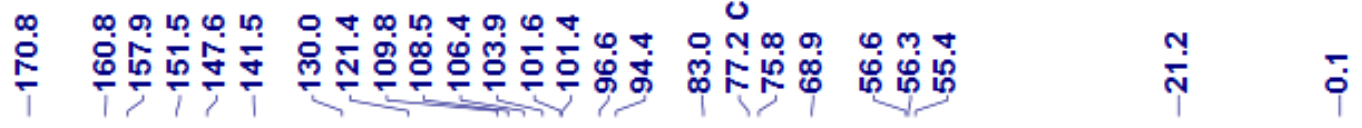<smiles>COc1cc2c(cc1[C@@H]1OC[C@@H]3CO[C@@]3(OC(C)=O)[C@H]1OC(C)=O)OCO2</smiles>

$( \pm)-31$ a

${ }^{13} \mathrm{C}$ NMR

(101 MHz, $\left.\mathrm{CDCl}_{3} / \mathrm{TMS}\right)$

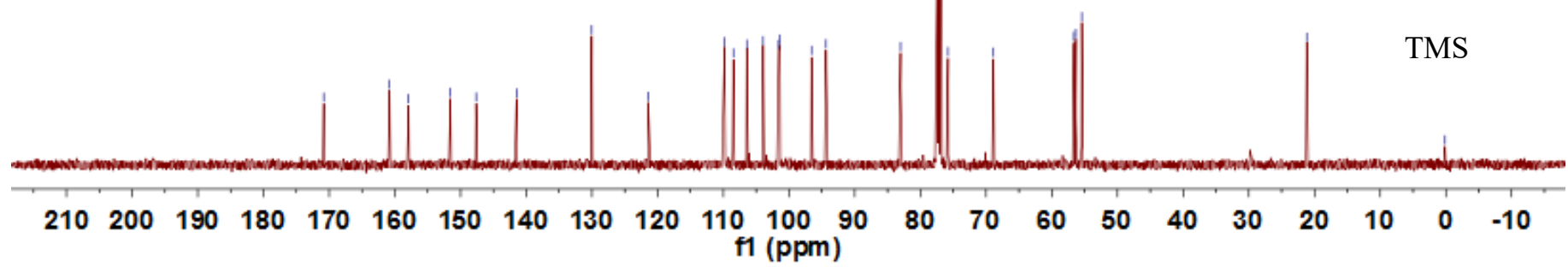




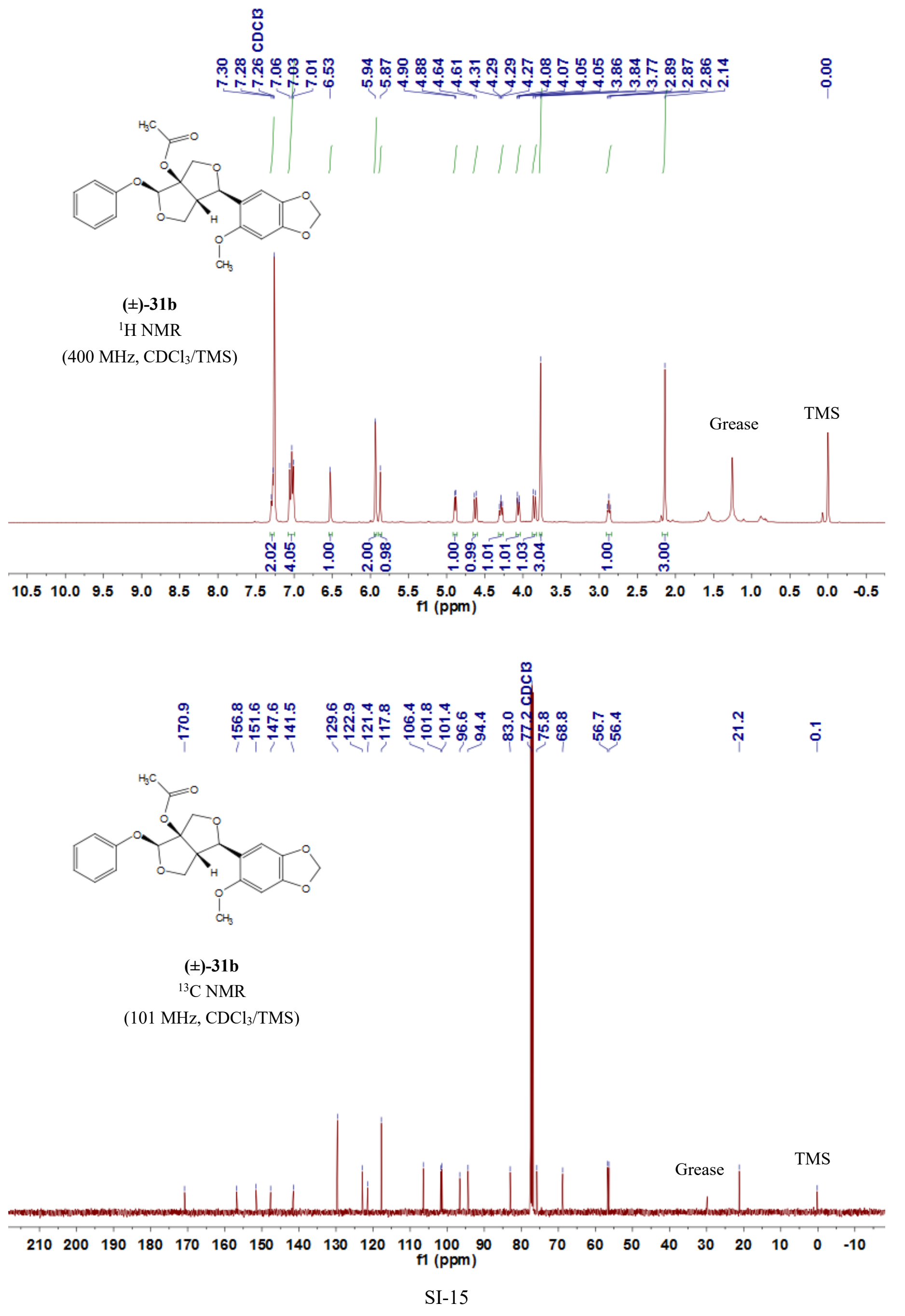


응

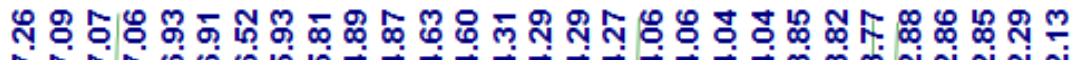

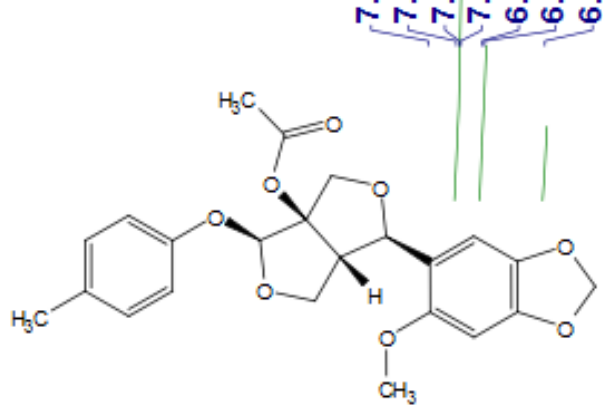

$\stackrel{8}{\circ}$

( \pm -31c

${ }^{1} \mathrm{H}$ NMR

(400 MHz, $\mathrm{CDCl}_{3} / \mathrm{TMS}$ )
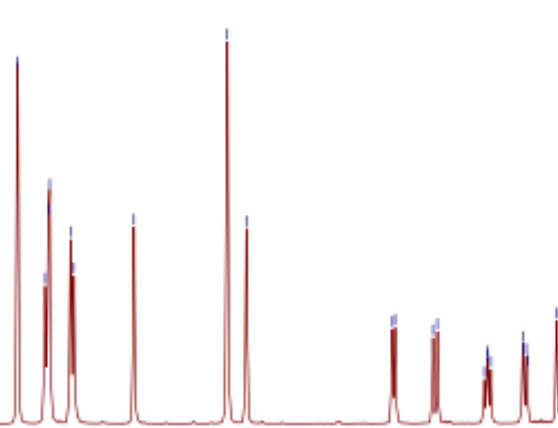

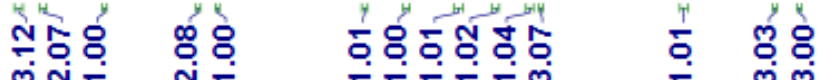

$\begin{array}{lllllllllllllllllllllll}10.5 & 10.0 & 9.5 & 9.0 & 8.5 & 8.0 & 7.5 & 7.0 & 6.5 & 6.0 & 5.5 & 5.0 & 4.5 & 4.0 & 3.5 & 3.0 & 2.5 & 2.0 & 1.5 & 1.0 & 0.5 & 0.0 & -0.5\end{array}$

$\stackrel{m}{0}$

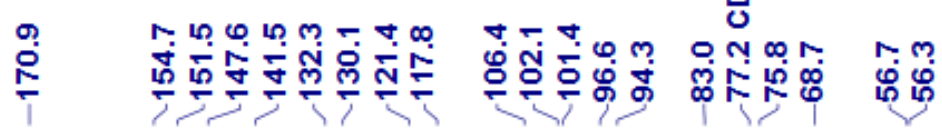

ஸุ

$\mathrm{H}_{3} \mathrm{C}$

$p$

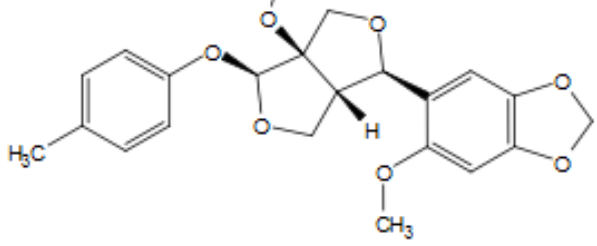

( \pm -31c

${ }^{13} \mathrm{C}$ NMR

(101 MHz, $\mathrm{CDCl}_{3} / \mathrm{TMS}$ )

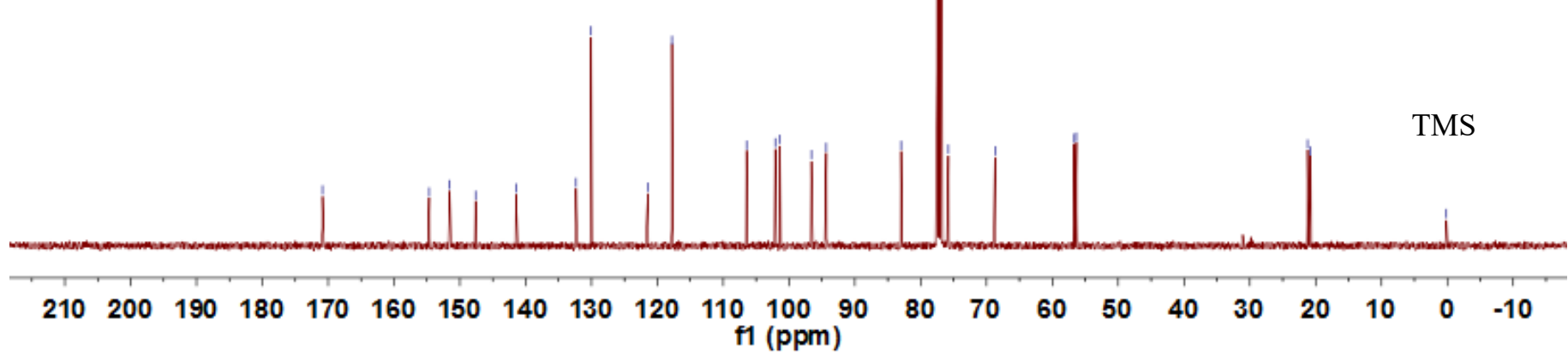


음

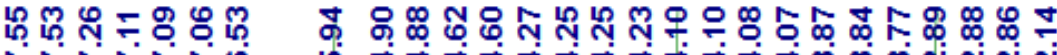

Ninko

ம0

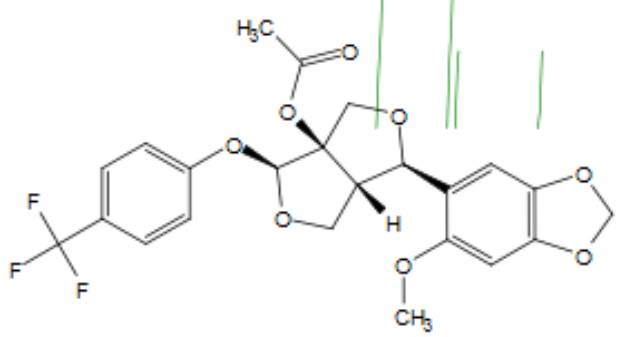

TMS

$( \pm)-31 d$

${ }^{1} \mathrm{H}$ NMR

(400 MHz, $\left.\mathrm{CDCl}_{3} / \mathrm{TMS}\right)$
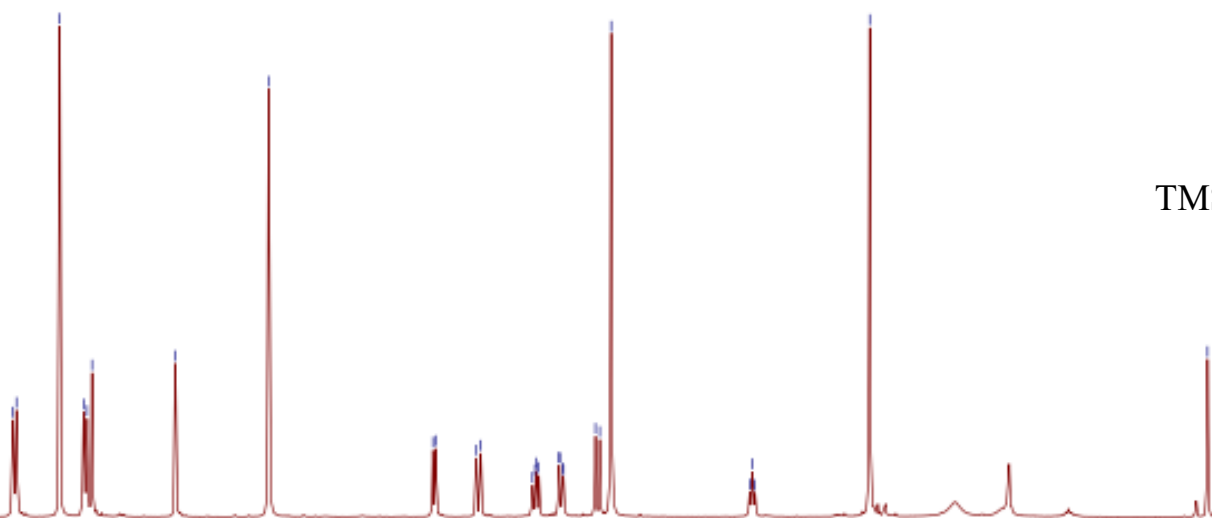

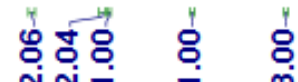

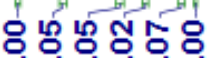

$\stackrel{1}{8} \stackrel{8}{-}$

$\begin{array}{lllllllllllllllllllllll}10.5 & 10.0 & 9.5 & 9.0 & 8.5 & 8.0 & 7.5 & 7.0 & 6.5 & 6.0 & 5.5 & 5.0 & 4.5 & 4.0 & 3.5 & 3.0 & 2.5 & 2.0 & 1.5 & 1.0 & 0.5 & 0.0 & -0.5\end{array}$

ํㅗㅁ

实

은 돈

$\stackrel{\bar{\tau}}{\bar{i}}$
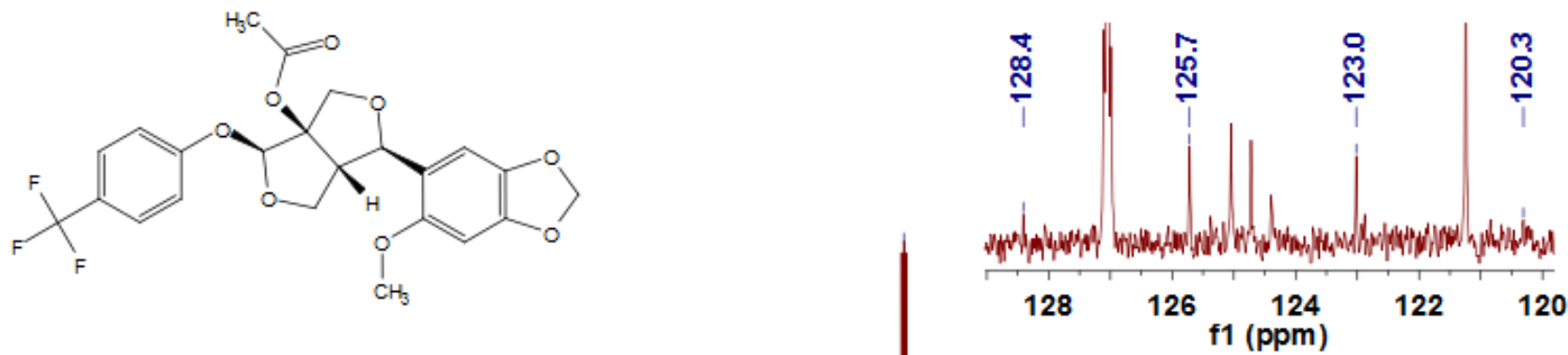

( \pm )-31d

${ }^{13} \mathrm{C}$ NMR

(101 MHz, $\mathrm{CDCl}_{3} / \mathrm{TMS}$ )

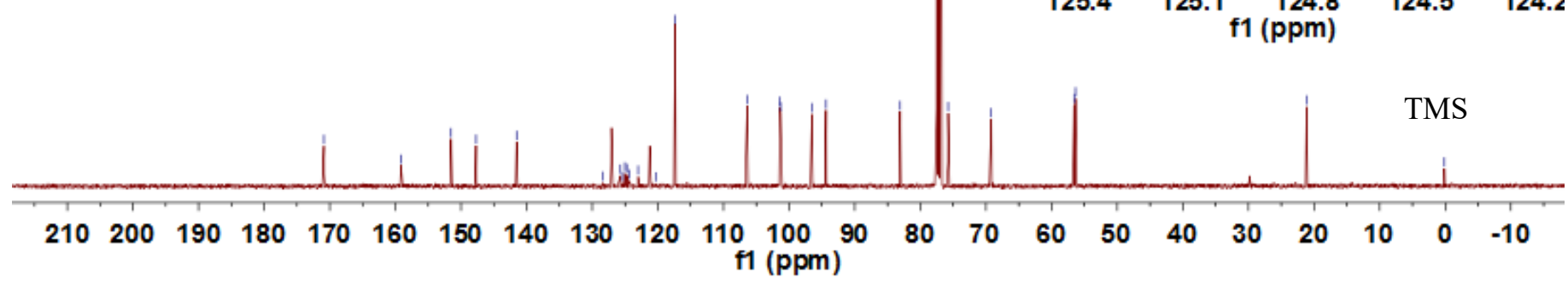


임

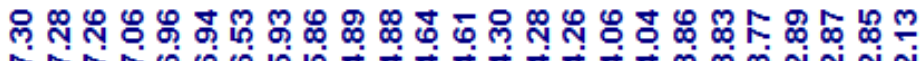

NNN

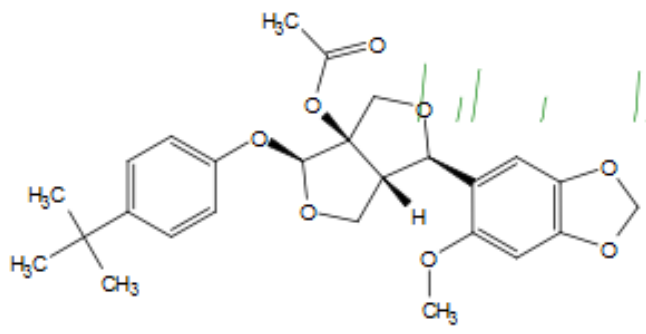

( \pm -31e

${ }^{1} \mathrm{H}$ NMR

(400 MHz, $\left.\mathrm{CDCl}_{3} / \mathrm{TMS}\right)$

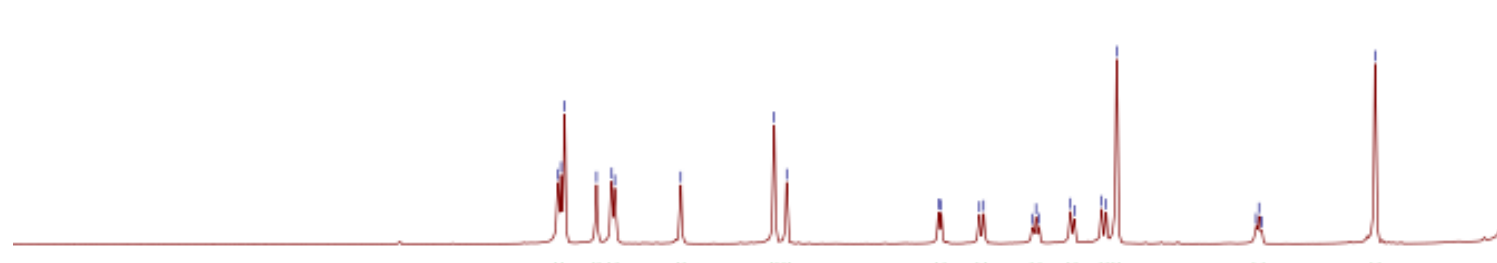

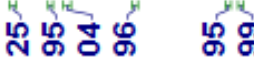

กั่

$\begin{array}{lllllllllllllllllllllll}10.5 & 10.0 & 9.5 & 9.0 & 8.5 & 8.0 & 7.5 & 7.0 & 6.5 & 6.0 & 5.5 & 5.0 & 4.5 & 4.0 & 3.5 & 3.0 & 2.5 & 2.0 & 1.5 & 1.0 & 0.5 & 0.0 & -0.5\end{array}$ $\mathrm{f1}(\mathrm{ppm})$

응

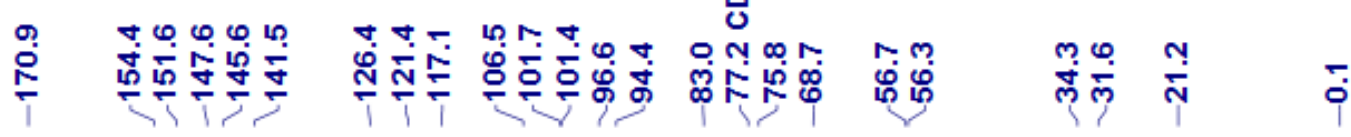<smiles>COc1cc2c(cc1[C@@H]1OC[C@@]3(OC(C)=O)[C@H](Oc4ccc(C(C)(C)C)cc4)OC[C@H]13)OCO2</smiles>

$( \pm)-31 \mathrm{e}$

${ }^{13} \mathrm{C}$ NMR

$\left(101 \mathrm{MHz}, \mathrm{CDCl}_{3} / \mathrm{TMS}\right)$

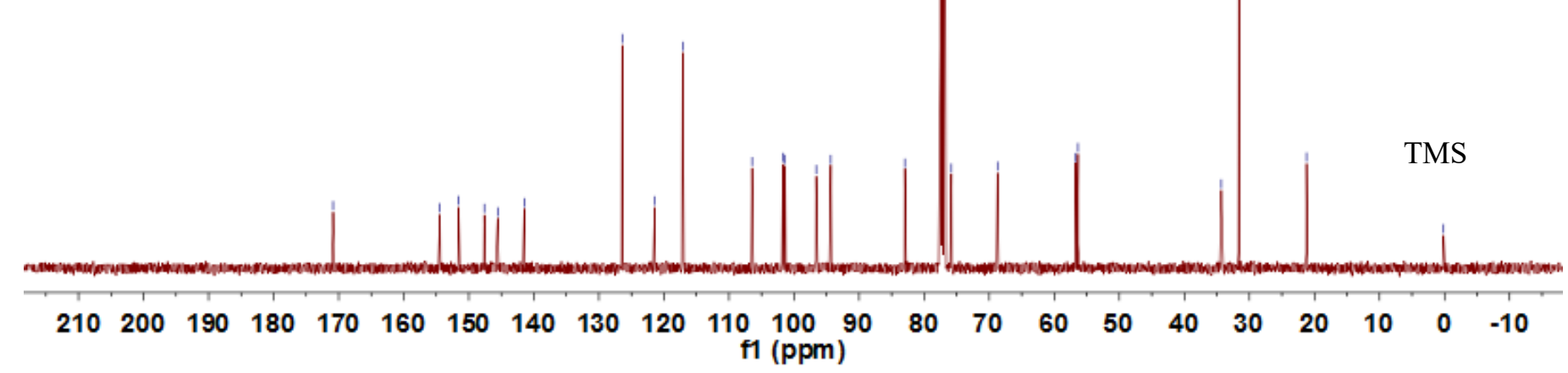


骨 ஸ̣̆

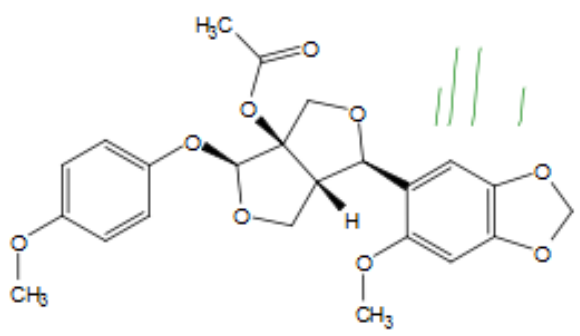

(土)-31f

${ }^{1} \mathrm{H}$ NMR

(400 MHz, $\mathrm{CDCl}_{3} / \mathrm{TMS}$ )

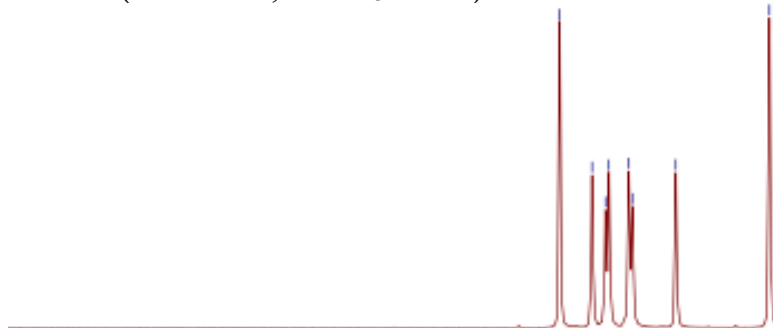

TMS

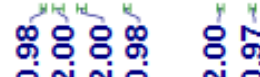

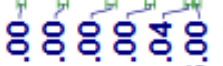

管

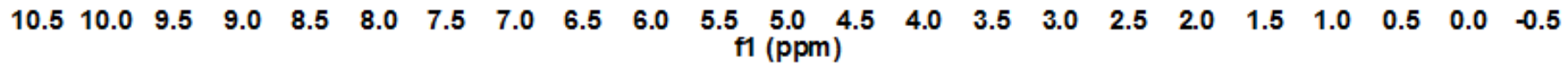

号

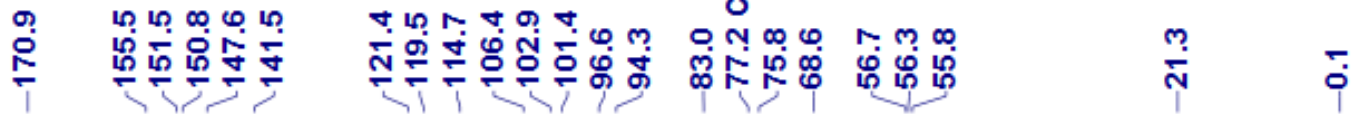<smiles>COc1ccc(O[C@@H]2OC[C@H]3[C@H](c4cc5c(cc4OC)OCO5)OC[C@@]23OC(C)=O)cc1</smiles>

(土)-31f

${ }^{13} \mathrm{C}$ NMR

(101 $\left.\mathrm{MHz}, \mathrm{CDCl}_{3} / \mathrm{TMS}\right)$

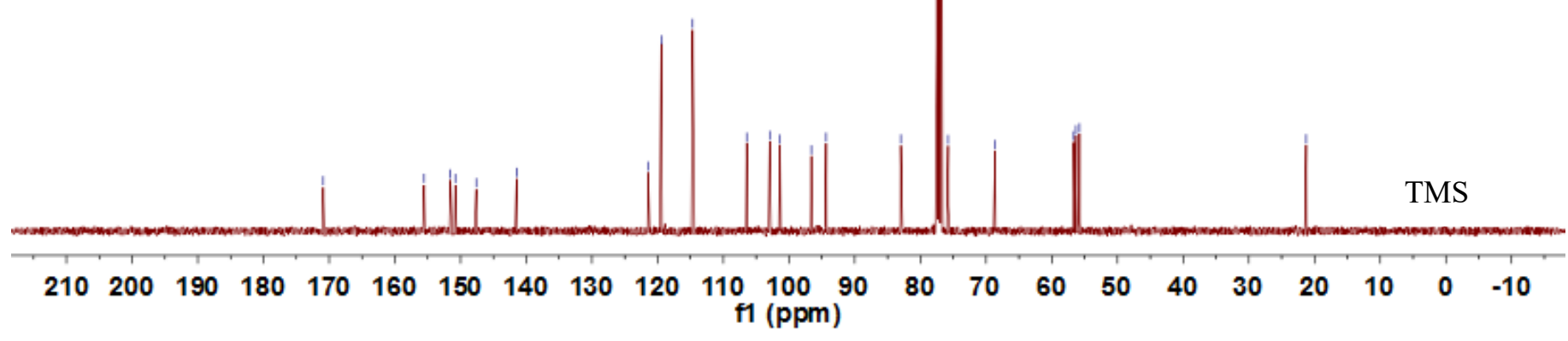




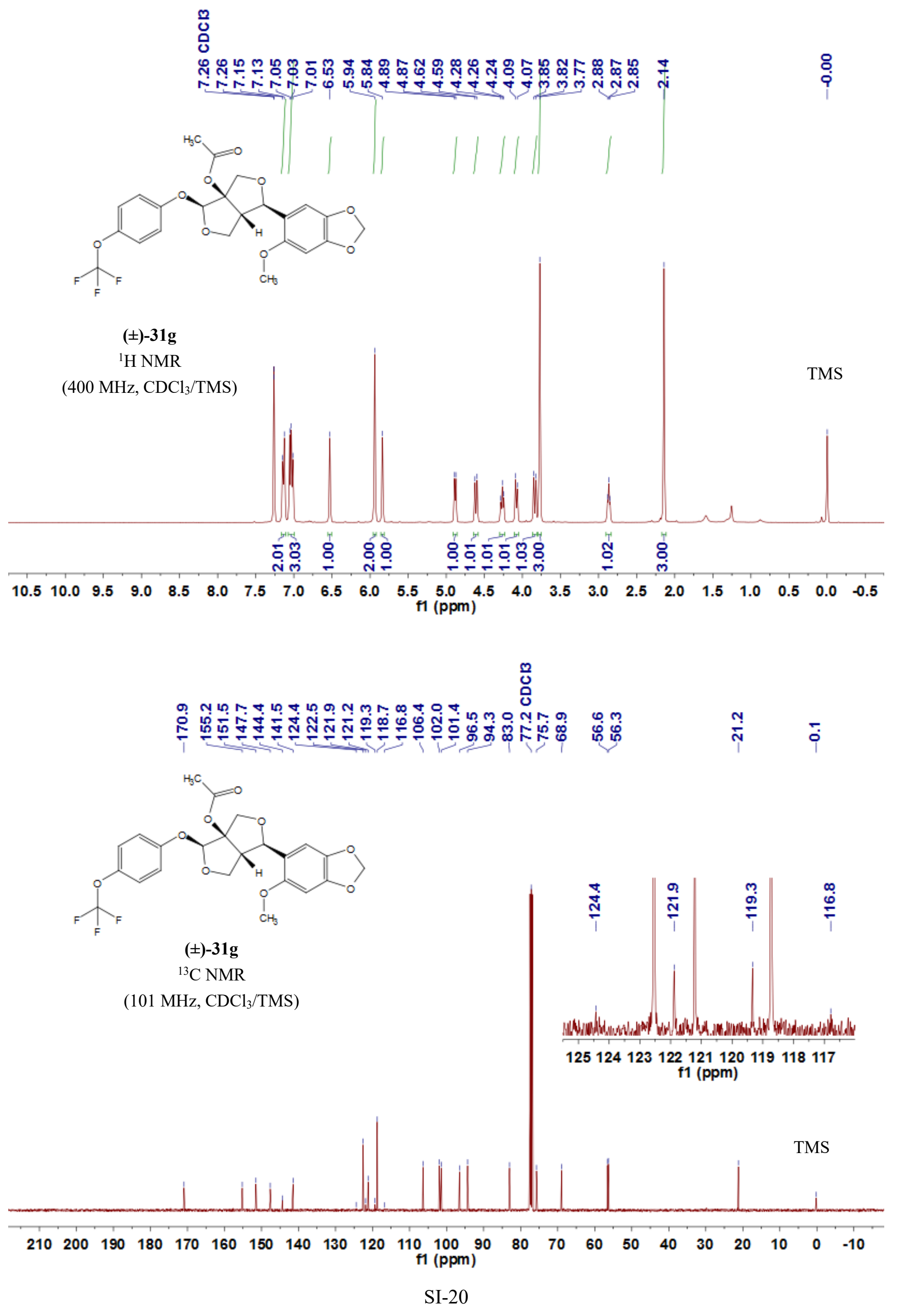




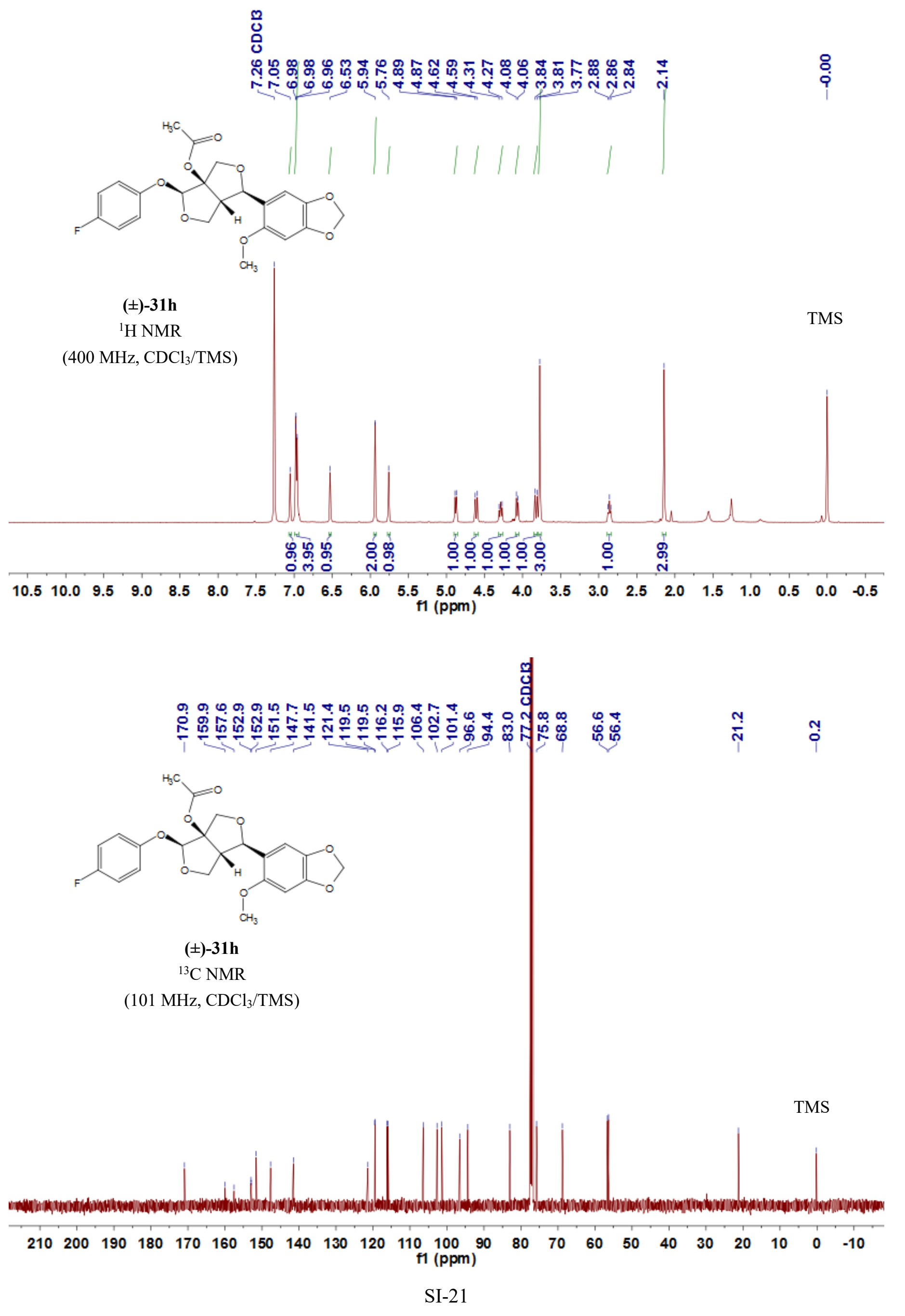


응

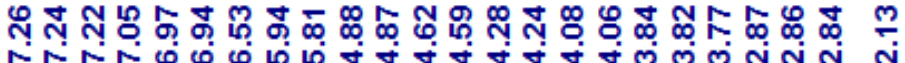
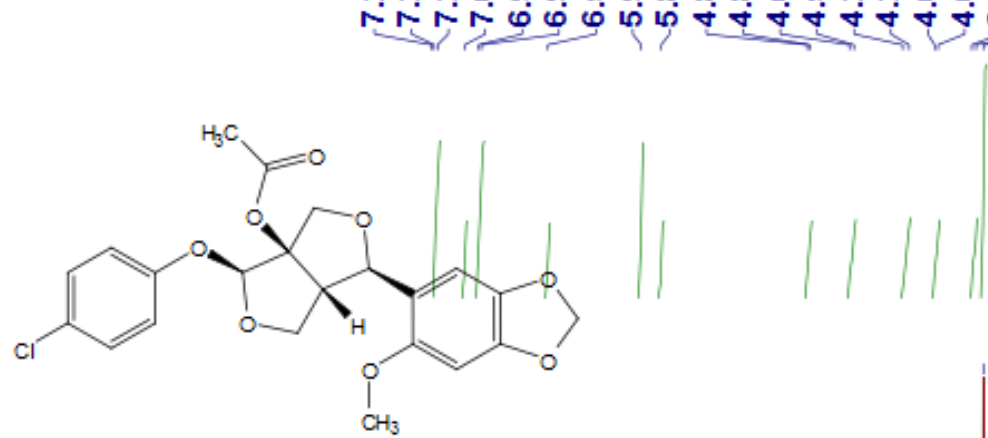

$( \pm)-31 i$

${ }^{1} \mathrm{H}$ NMR

(400 MHz, $\left.\mathrm{CDCl}_{3} / \mathrm{TMS}\right)$
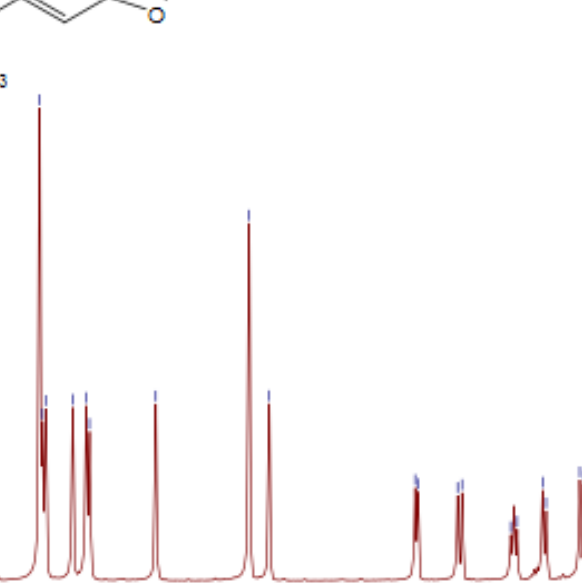

TMS

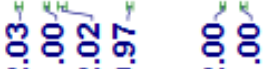

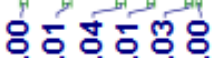

管

$\begin{array}{lllllllllllllllllllllll}10.5 & 10.0 & 9.5 & 9.0 & 8.5 & 8.0 & 7.5 & 7.0 & 6.5 & 6.0 & 5.5 & 5.0 & 4.5 & 4.0 & 3.5 & 3.0 & 2.5 & 2.0 & 1.5 & 1.0 & 0.5 & 0.0 & -0.5\end{array}$ $11(\mathrm{ppm})$

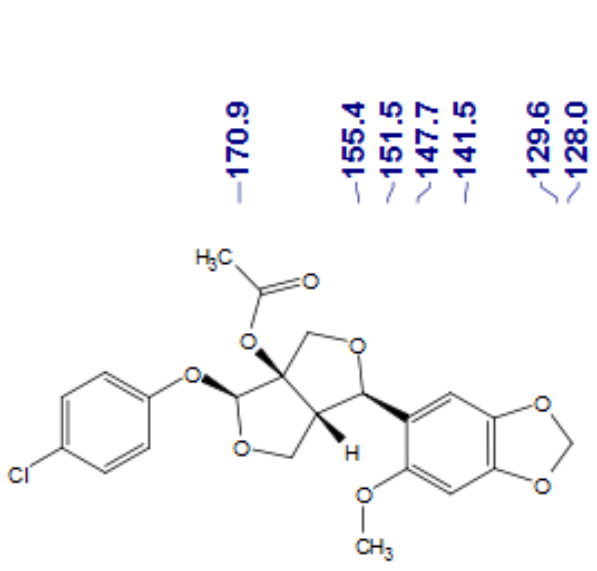

$( \pm)-31 i$

${ }^{13} \mathrm{C}$ NMR

$\left(101 \mathrm{MHz}, \mathrm{CDCl}_{3} / \mathrm{TMS}\right)$

वैं

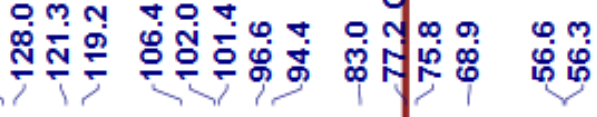

$\stackrel{\text { ஸุ }}{\overline{0}}$

TMS 
옹

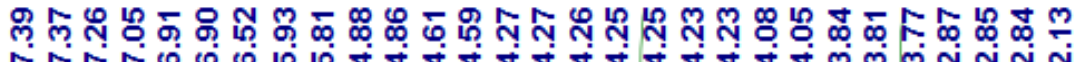

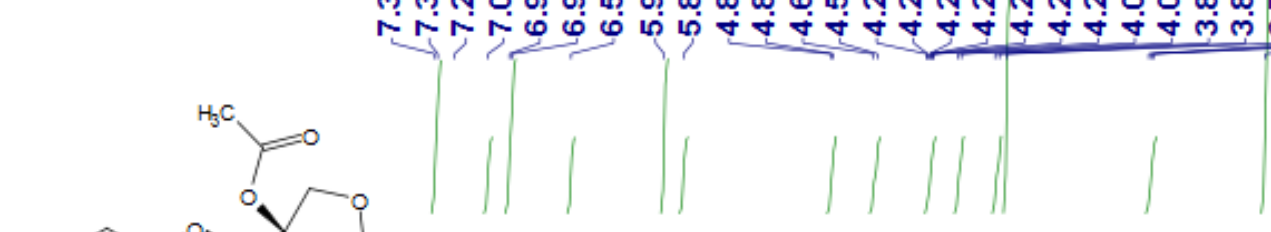<smiles>C[C@]1(F)CO[C@H](O)C1</smiles><smiles>Cc1cc2c(cc1C)OCO2</smiles>

(土)-31j

${ }^{1} \mathrm{H} \mathrm{NMR}$

(400 MHz, $\mathrm{CDCl}_{3} / \mathrm{TMS}$ )

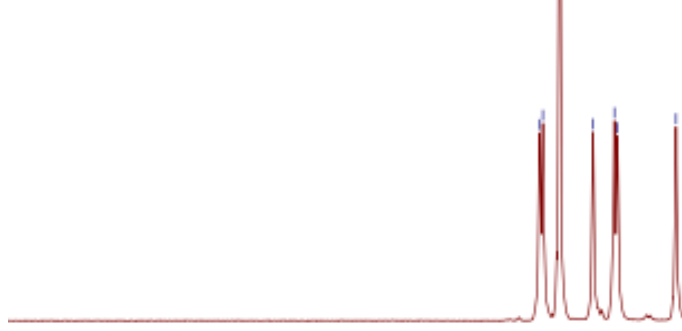

80

ํㅗㅇำ

\section{웅후유유}

$\stackrel{8}{8}$

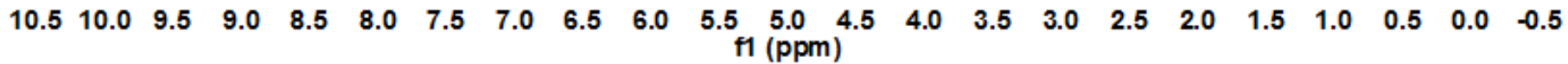

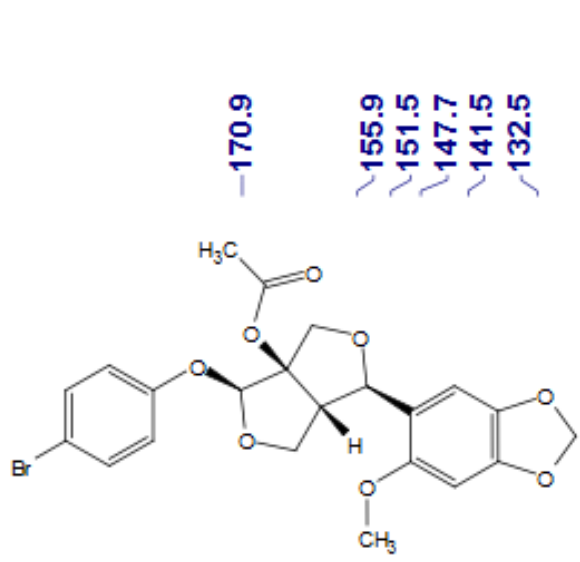

$( \pm)-31 j$

${ }^{13} \mathrm{C}$ NMR

(101 MHz, $\mathrm{CDCl}_{3} / \mathrm{TMS}$ ) 

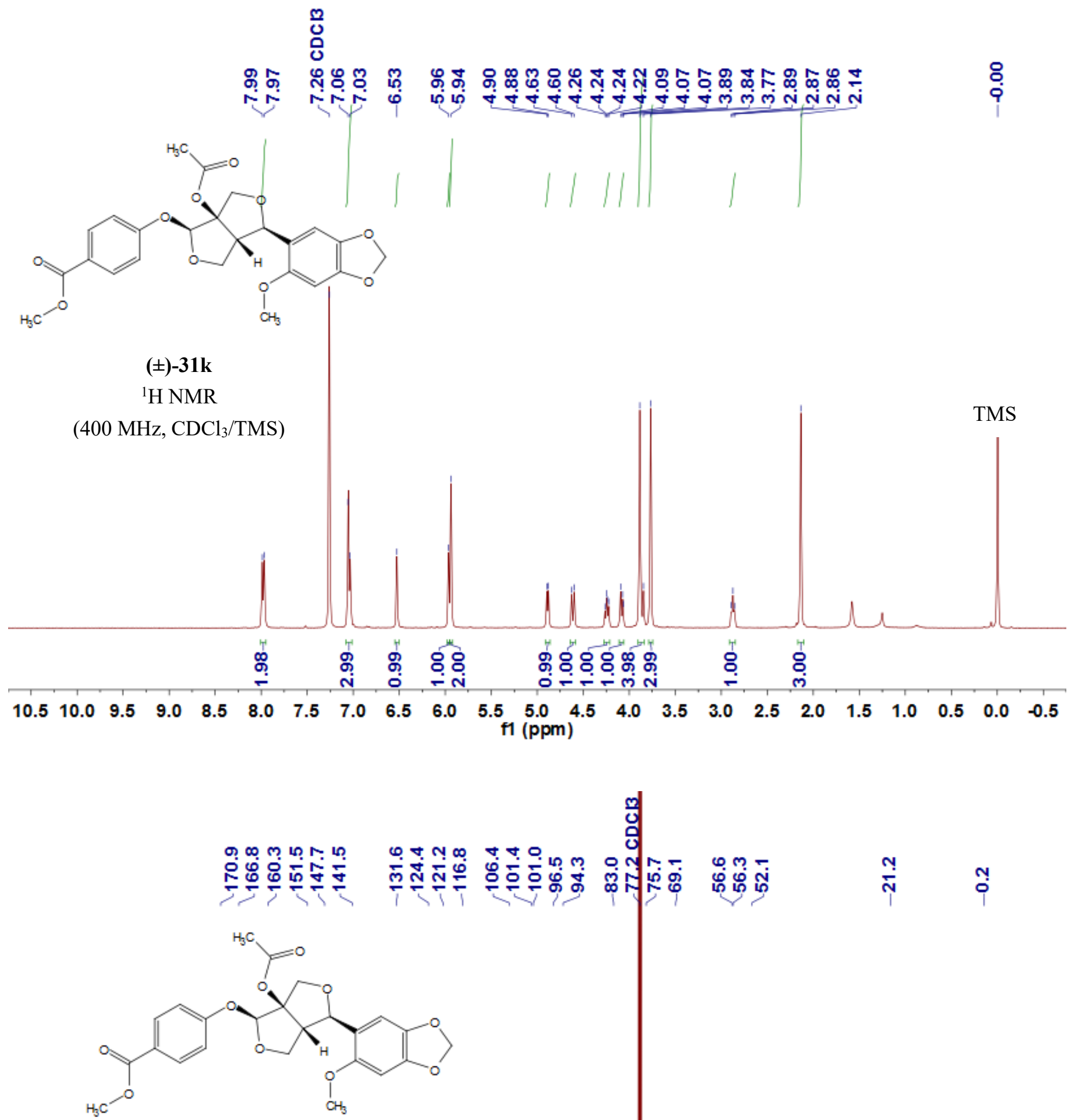

$( \pm)-31 k$

${ }^{13} \mathrm{C}$ NMR

(101 MHz, $\left.\mathrm{CDCl}_{3} / \mathrm{TMS}\right)$

웜
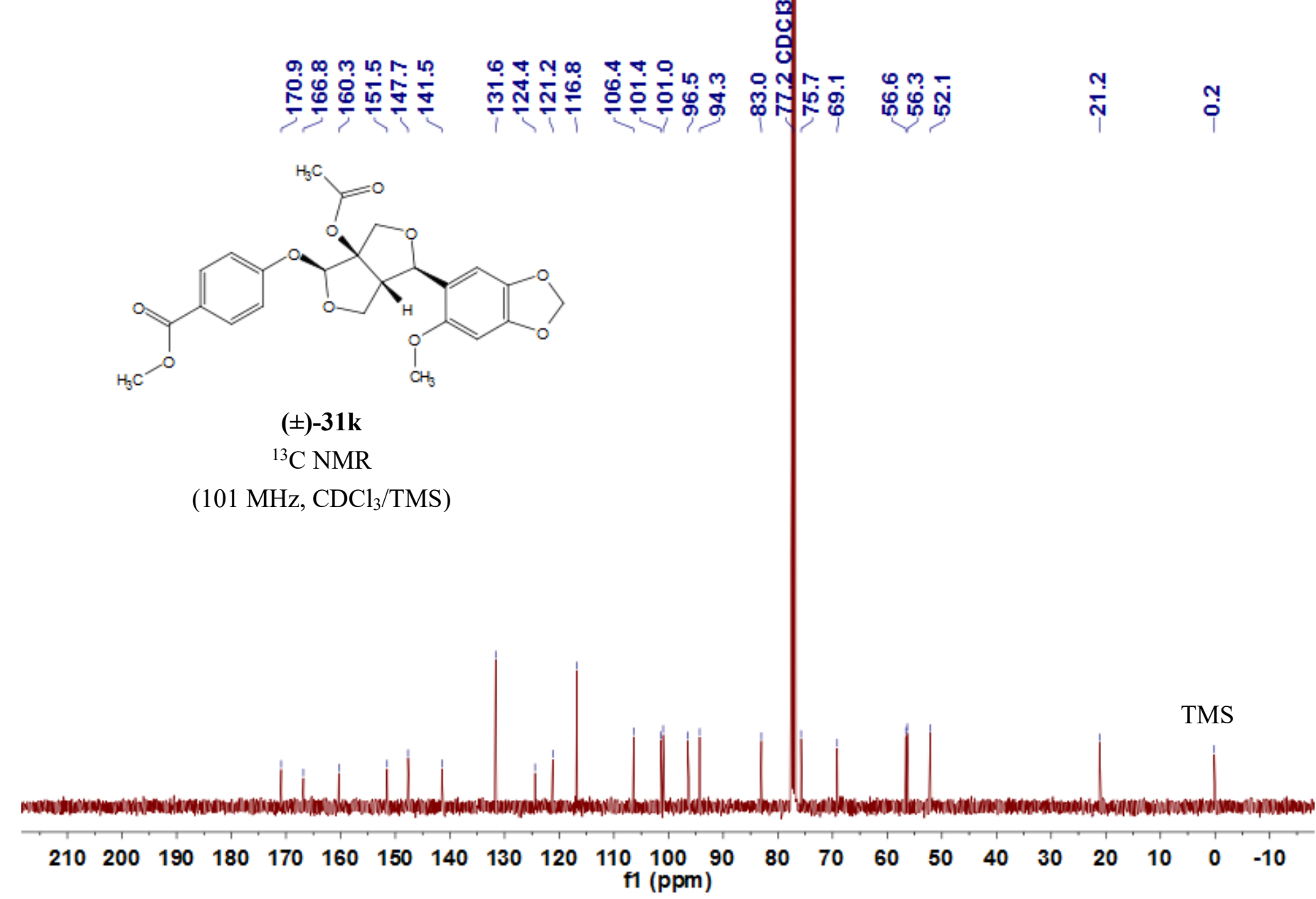